\title{
DIE ,NEUE' LEHRERBILDUNG IN DEUTSCHLAND. VOM PRAXISSCHOCK ZUR AUFGEKLÄRTEN ANEIGNUNG PROFESSIONELLEN HANDELNS? - LEHRERBILDUNG ALS SYSTEMISCHER TEIL DER BILDUNGSREFORM
}

\author{
THE 'NEW' INITIAL TEACHER EDUCATION IN GERMANY. FROM SHOCK IN PRACTICE TO \\ ENLIGHTENED ADOPTION OF PROFESSIONAL PRACTICE? \\ TEACHER EDUCATION AS SYSTEMIC PART OF THE GERMAN EDUCATIONAL REFORM
}

\author{
JÜRGEN VAN BUER'; KATHRIN PETZOLD-RUdOLPH \\ Humboldt-Universität zu Berlin (Deutschland)
}

\section{ZUSAMMENFASSUNG}

Seit über zehn Jahren ist die Lehrerbildung in Deutschland in den Bologna Prozess integriert. In der Folge wurde das Studienmodell mit 1. Staatsexamen und anschließendem 2. Staatsexamen zum Abschluss des Vorbereitungsdienstes für den Bereich des Hochschulstudiums auf die Bachelor-Master-Struktur umgestellt. Aktuell wird die zweite Runde der Studienprogramm-Reform implementiert. Diese fokussiert vorrangig die erziehungswissenschaftlichen und die fachdidaktischen Module. Dabei stellt sich durchaus drängend die Frage, wie Konzepte erfahrungsgebundenen feldorientierten Lernens verstärkt in das Studium des Lehramtes eingebunden werden können. Als eine Konsequenz flammen die durchaus kontrovers geführten Debatten über das Verhältnis von 'Theorie' und 'Praxis' in der Lehrerbildung erneut auf. Der Vorwurf der Befürworter erfahrungsbasierter Lernkonzepte lautet, die traditionelle Sichtweise der Lehrerbildung sei als weitgehend gescheitert anzusehen und durch ein Modell zu ersetzen, welches berufliche Kompetenzen zugrunde legt, die sowohl differenzierte wissenschaftlich fundierte Wissensstände als auch verhaltensbasierte Lehrstrategien und Fähigkeiten umfassen. Der folgende Artikel skizziert diese widerstreitenden Diskussionen - aus einer eher theoretischen Sichtweise wie auch aus der kritischen Betrachtung der einschlägigen empirischen Befunde. Dabei wird die Notwendigkeit sichtbar auszuführen, in welchem Ausmaß die aktuellen Reformen in der Lehrerbildung als systemischer Bestandteil des Gesamtkonzepts der Erneuerung des deutschen Schulwesens ausgestaltet werden.

Palabras clave: Evidenzbasierte Bildungspolitik; Theorie und Praxis in der universitären Lehrerbildung; Schulpraktika in der universitären Lehrerbildung; Strukturen; Empirische Ergebnisse; Universitäre Lehrerbildung und Qualitätsentwicklung von Schule; Reform der deutschen Lehrerbildung.

\begin{abstract}
Since about ten years initial teacher education in Germany has been integrated into the Bologna Process. Subsequently the 1 rst state exam-model has been transformed into study programs beginning at the Bachelor level and including the Master of Education; afterwards, the successful student begins the second part of his professional education within the state dominated internship. Actually, the second round of study program reforms is on the way of implementation. The reforms focus mainly the pedagogical modules and those of special didactics. Subsequently the question how to strengthen field-based learning is one of the most significant requirements. In consequence the question of 'theory' and 'practice' in initial teacher education gets back into the still controversial debates. The reproval of the field-based proponents is that the traditional view on teacher education has failed and has to be substituted by an underlying model of professional competences including differentiated knowledge as well as behavioral teaching strategies and
\end{abstract}

${ }^{1}$ Corresponding author: Jürgen van Buer. E -mail: van.buer@rz.hu-berlin.de 
skills. The following article outlines these conflicting discussions, as well from a more theoretical point of view as from a critical view on the empirical results. For an adequate understanding of these positions it is worthwhile to know that the actual reforms in German teacher education are arranged as a systemic part of the general reforming processes in the educational system. Therefore, the interaction between these two fields of transformation are discussed, too.

Key words: Evidence based Policy in Education; Theory and practice in initial teacher education; School internship in initial teacher education; Structures; Empirical findings; Initial teacher education and school enhancement; Reform in German initial teacher education. 


\section{Zur Einleitung - „Neue Lehrer braucht das Land“}

"Neue Lehrer braucht das Land“. So oder so ähnlich lautet in den letzten Jahren eine ganze Reihe von Überschriften aus der täglichen Presse oder Wochenjournals (vgl. z. B. ZEIT Online, 05.01.2005; stern, 03.11.2008; Hauptsache Bildung, 06.06.2014). Wenn man die teils massiven Veränderungen hinsichtlich der Steuerung des Schulsystems, in der Binnenstruktur der Einzelschulen sowie bezüglich der Anforderungen an das unterrichtliche Handeln der Lehrkräfte ihn Betracht zieht, sind die Forderungen, die in dem obigen Slogan thematisiert werden, durchaus nachvollziehbar. Sie zielen vor allem darauf, die Nutzbarkeit des Wissens, das in der universitären Lehrerbildung den späteren Lehrkräften vermittelt wird, für die Problemlösung vor Ort in Schule und Unterricht zu steigern. Als Symbol für diese durchaus problembeladene Kopplung von akademischem Wissen und dessen direkter Handlungsnutzung steht in Deutschland für die Lehrerbildung das Wort vom „Praxisschock“; damit wird das folgende Phänomen gekennzeichnet: Bei ihrem Antritt als Lehrkraft benötigen die noch unerfahrenen Lehrkräfte bei all ihrer guten Ausbildung in den Fachwissenschaften wie auch in der Fachdidaktik bzw. Erziehungswissenschaften für das alltägliche kompetente Lösen von Problemen in Schule und Unterricht eine Vielzahl zusätzlicher, während ihrer Ausbildung nicht trainierter Kompetenzen; gleichzeitig erfahren sie, dass sie dafür das in der Universität Gelehrte nur begrenzt nutzen können. Diesen Praxisschock mildern bzw. gänzlich überwinden zu helfen, ist ein wichtiges Ziel der Reform der Lehrerbildung.

Gleichzeitig macht die Altersverteilung der beschäftigten Lehrerkräfte in Deutschland auf erhebliche Rekrutierungsprobleme aufmerksam (vgl. KMK 2011a); denn in einzelnen Schulformen scheidet in den nächsten Jahren bundesweit ein Großteil aus und muss ersetzt werden:

- So sind in sechs der sechszehn Bundesländern mehr als 50\% aller Lehrkräfte älter als 50 Jahre; davon sind ca. $10 \%$ älter als 60 Jahre.

- Zudem werden Lehrkräfte vor allem aufgrund gesundheitlicher Belastung im Durchschnitt mit ca. 63 Jahren pensioniert.

- In der Folge von Dienstalter und Belastung wurden im Jahr 2012 insgesamt 24.400 Lehrer und Lehrerinnen in den Ruhestand versetzt (vgl. Spiegel online, Schulspiegel, 24.01.2014).

Vor diesem Hintergrund ist es nur folgerichtig, die Modernisierung der Professionalität des Lehrpersonals nicht nur über Angebote zur beruflichen Weiterbildung zu erreichen, sondern bereits früh bei der Berufsausbildung der kommenden Lehrkräfte anzusetzen. In Deutschland sind dies die universitären Studienprogramme einerseits und die Ausbildung im Vorbereitungsdienst (Referendariat) andererseits (zur Struktur der deutschen Lehrerbildung vgl. Abschnitt 3.2).

Angesichts des oben Skizzierten ist es nicht überraschend: Die Berufsausbildung zur Lehrerkraft an einer öffentlichen Schule erfährt in Deutschland seit Jahren erhöhte Aufmerksamkeit; dies gilt in besonderem Maße für die erste Stufe, die universitäre Lehrerbildung: So veranstaltete z. B. die Deutsche Telekom Stiftung gemeinsam mit dem Stifterverband für die Deutsche Wissenschaft und mit der Hochschulrektorenkonferenz (HRK) 2015 eine viel beachtete Tagung zu dem Thema "Lehrerbildung Verantwortung für die Zukunft. Herausforderungen und Perspektiven" (vgl. Deutsche Telekom Stiftung 2015). Dabei ging es zum einen um eine kritische Bestandsaufnahme; zum anderen ging es um die Definition von Entwicklungsoptionen für die Ausbildung. Unterstützt wird diese Debatte durch zusätzliche Förderungen, die auf die Erhöhung der faktischen Qualität der universitären Lehrerbildung zielen; so werden für das auf 10 Jahre hin angelegte Programm „Qualitätsoffensive Lehrerbildung" bis zu 500 Millionen Euro zusätzlich zur Verfügung gestellt (Bund-Länder-Vereinbarung 2013).

\section{Schule und Unterricht - zum Spannungsverhältnis von tradiertem geisteswissenschaftlichem Verständnis und aktuellen bildungspolitischen Effizienzforderungen}

Durch die wenig zufriedenstellenden Ergebnisse der PISA-Studien hinsichtlich der schulischen Lernoutputs in der Sekundarstufe I des deutschen Schulwesens (vgl. z. B. Ehmke, Klieme \& Stanat 2013) wurde in Deutschland der so genannte PISA-Schock ausgelöst; in der Folge werden in den deutschen Bundesländern seit ca. 15 Jahren tief greifende Veränderungen bezüglich der Steuerung des Schulwesens angestrebt. Die Veränderung der Steuerungsphilosophie reicht hinein bis in die strategische und operative 
Organisation der einzelschulischen Qualitätsentwicklungsprozesse, hier vor allem bezüglich des Unterrichts. Allerdings sind aufgrund der strikt föderalen Struktur der Bundesrepublik gerade für das Bildungswesen (vgl. Rürup 2005) im Vergleich der 16 Bundesländer deutliche Unterschiede feststellbar. Gleichwohl wird eine durchaus vergleichbare Ausrichtung der Veränderungsprozesse sichtbar; diese betrifft vor allem die Generierung der Instrumente, die in der Qualitätsentwicklung eingesetzt werden (sollen) (z. B. das Instrument der Schulinspektion als einem Instrument der externen Evaluierung; vgl. z. B. van Buer, Wagner, Rückmann \& Füssel 2015).

Um ein detaillierteres Verständnis bezüglich der jüngsten Reformen in der deutschen Lehrerbildung entwickeln zu können, liegt es nahe, zumindest die beiden folgenden Kontexte näher zu betrachten: Zum einen geht es um die Frage, inwiefern das Grundverständnis von Unterricht als einen durch Widersprüche gekennzeichneten Handlungs- und Entscheidungsraum und damit dem tradierten Verständnis von , Theorie und ,Praxis' auch weiterhin Gültigkeit beigemessen wird (vgl. Abschnitte 2.1 und 2.2); zum anderen geht es um die bildungspolitisch induzierte outputorientierte Steuerung von Schulsystem, Schule und Unterricht als Leitfigur von Professionalität (vgl. Abschnitt 2.2.1).

\subsection{Zu Schule und Unterricht als ill defined problem}

\subsubsection{Schule und Unterricht als Räume multiperspektivischer und konfligierender Steuerung}

Für Maroy \& van Zanten (2009: 69) beinhaltet die Steuerung von Schule zu wesentlichen Teilen „multiple, contradictory and sometimes conflicting processes for orienting the behaviors or factors and defining the rules of the game in a social system". So machen Governance-Konzepte auf die multiperspektivische, in Teilen auch konfligierende soziale Regulation von Steuerung, Koordination und Aushandlung in Schule und Unterricht aufmerksam: Folgt man diesen Konzepten, geht es darum, die interdependenten Handlungen sozialer Akteure in institutionellen Rahmenbedingungen auf der Basis von Aushandlungsprozessen zu koordinieren (z. B. Heinrich 2008). Beachtet werden sollten dabei auch die „partial and fragile interarticulation of various forms of institutional co-ordination“ (Dupriez \& Maroy 2003: 379). Besonders die Koordinierung, die mehrere hierarchische Ebenen umfasst, führe zu folgendem Sachverhalt: Die Akteure kontextualisieren die vereinbarten Regularien; sie interpretieren diese „on the microlevel of the day-to-day work" (de Boer, Enders \& Schimank 2007: 150), und sie reinterpretieren diese Regularien Tag für Tag zumindest in Teilen jeweils aufs Neue. Die derzeitig favorisierte starke Betonung von output-orientierter Steuerung (z. B. Böttcher 2006) führt zur Stärkung von interner und externer Evaluation und damit zur Favorisierung von daten- und ergebnisgestützter evidenz-basierter Schulpolitik (vgl. Abschnitt 2.2.1). Für Schule bedeutet dieses Konzept nicht zuletzt die Verstärkung messtechnisch untermauerter Outputerfassung (z. B. van Ackeren \& Brauckmann 2010; kritisch Heinrich 2010). Damit werden die Widersprüche und Inkonsistenzen von Allokation, Selektion und Support tendenziell verschärft, die für Schule aus geisteswissenschaftlicher Perspektive bereits in den 1990er Jahren differenziert diskutiert wurden.

\subsubsection{Handlungsparadoxa als unausweichliche Merkmale unterrichtlicher Lehrtätigkeit}

Folgt man Helsper (1996), ist unterrichtliches Handeln von Lehrkräften in der öffentlichen Institution Schule grundlegend gekennzeichnet durch Paradoxa. Diese seien den dortigen Akteuren nicht immer bewusst; allerdings intensivierten sie deren Gefahr, in „routinisierte Erstarrung“ (Helsper 1996: 535) zu verfallen. Helsper (1996: 537f.) identifiziert verschiedene grundlegende pädagogische Handlungsparadoxa; diese würden durch die Modernisierungsprozesse in Gesellschaft gleichsam als nicht-intendierte ,Folgekosten' für Schule und Unterricht generiert; und sie zeigen sich - so könnte man betonen - auch aktuell in Schule und Unterricht (z. B. zur Bildungsbeteiligung von Migranten und Migrantinnen vgl. z. B. die Beiträge in Auernheimer 2006; zum inklusiven Unterricht vgl. z. B. KMK 2015; kritisch Ahrbeck 2014). Schaut man auf die aktuelle Favorisierung evidenz-basierter Schulpolitik und auf die unübersehbaren Tendenzen zur Normierung von Schule und Unterricht über die Definition von Standards (vgl. Abschnitt 2.2.2), spielt das Individualisierungsparadoxon eine besondere Rolle: Dieses thematisiert den Widerspruch, der in der "Vieldeutigkeit der Sichtweisen sozialer und subjektiver Welt" (Helsper 1996: 541) gerade auch für Schule sichtbar wird. Denn diese Vieldeutigkeit werde vor allem für die im Unterricht zu verhandelnden 
Die ,neue' Lehrerbildung in Deutschland. Vom Praxisschock zur aufgeklärten Aneignung professionellen Handelns?...

Gegenstände gesellschaftlich nicht nur legitimiert, sondern geradezu eingefordert. Diese Vielfalt von Sichtweisen bezieht sich auf die Perspektiven und Überzeugungssysteme, die die Schüler in Unterricht einbringen (vgl. dazu z. B. die Beiträge in Krüger, Deinert \& Zschach 2012), aber auch auf die Sichtweisen der Lehrer selbst. Diese Vielfalt stellt die Frage, inwiefern die durchaus erkennbare curriculare Normierung der Lernangebote (vgl. z. B. teaching for testing als Konsequenz; vgl. z. B. Popham 2001) dieses Individualisierungsparadoxon noch weiterhin verschärft.

\subsubsection{Unterricht als ill defined problem}

Folgt man den obigen Überlegungen, stellt sich geradezu folgerichtig die Frage nach der Entscheidungsund Handlungssicherheit, die durch professionelles Lehrhandeln in Unterricht erreichbar ist. Für Reinisch (2009: 34) besteht dieses Handeln aus „zwei in sich widersprüchlichen Elementen: Anwendung universeller Regeln und Verstehen des je besonderen Falls". Dies führe zu grundlegender Unsicherheit im Sinne von ill defined problems; diese - so Reinisch - stellen ein basales Merkmal der sozialen Konstruktion Unterricht dar. Diese Unsicherheit kann durch Handlungsregularien, wie sie etwa in der pädagogischen ,Meisterlehre und darauf basierenden Handbüchern für z. B. ,guten' Unterricht angeboten wird, nicht aufgelöst werden (als Beispiel für solche Handbücher vgl. z. B. Beyer 2014). Allerdings werde diese Unsicherheit immer wieder im day-to-day-work, überspielt' (de Boer, Enders \& Schimank 2007: 150); dies erfolge z. B. durch „routinierte Erstarrung" (Helsper 1996: 535). Eine andere Form zu versuchen, diese Unsicherheit zu begrenzen oder auch zu überspielen, kann in der kognitiv basierten Regulierung von Lehrhandeln im Sinne von Handlungskalkülen gesehen werden. Entgegen dieser letzteren gedanklichen Grundfigur zeigt Kurtz (2009: 46ff.): Auch durch systematische Erweiterung des professionellen Wissens wird für das Individuum das grundlegende Merkmal unterrichtlichen Handelns als ill defined problem nicht aufgelöst, sondern allenfalls partiell verringert. Denn in der prospektiven Begründung während des Planungshandelns bleiben die Entscheidungen durch „Situationsbezogenheit, (...) Verstehensdefizit und Komplexität der (erwarteten, hinzugefügt) Interaktion" unsicher (Kurtz 2009: 51). Auch bei der Realisierung von Unterricht ist grundlegend eine immer unvollständige Informationsbasis gegeben. In der Folge sind die jeweils getroffenen Entscheidungen letztlich erst in der retrospektiven Reflexion begründbar, auch die Entscheidungen zur Nutzung wissenschaftlich basierten Wissens. Zudem werde in dieser retrospektiven Begründung nachträglich eine stärkere Rationalität der Entscheidungs- und Handlungsstruktur reklamiert, als sie beim Planungsvorgang und dann vor allem während des Realisierungsprozesses faktisch vorhanden war. Folgt man diesem Gedanken, stellt sich durchaus die Frage, inwiefern erwartbar ist, dass durch die Ausweitung der Praxisphasen (vgl. Abschnitt 3.3.1) und die erhöhte Aneignung von praxisbezogenem Handlungswissen etc. im Sinne einer pädagogischen Meisterlehre der viel gefürchtete Praxisschock reduziert werden kann; bzw. es stellt sich die Frage, welche die Folgekosten etwa hinsichtlich des Erwerbs wissenschaftlich gesicherten Wissens über Schule und Unterricht sind (vgl. Abschnitt 3.3.3).

2.1.4. Theorie' und ,Praxis' und Professionalität des Unterrichtens - eine schier unendliche kontroverse Debatte?

Zur Einleitung: In der obigen Skizze deuten sich bereits die spannungsreichen Beziehungen von ,Theorie' als wissenschaftlich basierten und ,Praxis' als regulativen und/oder situational gebundenen Wissensstrukturen an (vgl. Abschnitt 2.1.5). Diese für den Handlungsalltag in Schule und Unterricht nach wie vor wenig geklärten Beziehungen, die für junge Lehrkräfte auch mit dem Begriff des Praxisschocks zusammengefasst werden, spiegeln sich ebenfalls in den Konzepten der deutschen Lehrerbildung: Zwar wird vor allem bildungspolitisch wie auch seitens der Akteure im staatlichen Vorbereitungsdienst (Referendariat) die Verstärkung der schulpraktischen Anteile gefordert; diese sollten zumindest der Intention nach zu einer neuen Ausbalancierung von ,Theorie' und ,Praxis' führen sollen. Die angestrebte Richtung zielt auf die stärkere Anlehnung an Konzepte erfahrungsgebundener feldorientierter Lehrerbildung (fieldbased teacher education; vgl. z. B. Zeichner \& Tabachnick 1981; Wisemann, Knight \& Cooner 2004; Zeichner 2010; für England vgl. Ertl 2015; Burstow 2015). Diese Konzepte, die sich vor allem im anglosaxonischen Raum schon seit Jahrzehnten etabliert haben, erreichen derzeit auch in Deutschland starke Aufmerksamkeit; damit entsteht dort eine historisch bereits kontroverse Debatte aufs Neue: Die Kritiker der oben angesprochenen Konzepte verstehen das Verhältnis von theorieorientiertem Lernen (college- bzw. 
university-based campus courses) und handlungspraktischem Lernen vor Ort in Schule (field-based setting) vor allem als ein Verhältnis, das nach wie vor ungeklärt und spannungsreich ist.

Zur Genese der Debatte zum Theorie-Praxis-Problem in Deutschland - eine Skizze: Die deutsche Debatte um das Theorie-Praxis-Problem kann auf eine lange Tradition zurückblicken (z. B. Werth 1985). Ein wichtiger Ausgangspunkt sind geisteswissenschaftliche Überlegungen zum Lehren in der öffentlichen Schule sowie zu den dort sichtbaren Antinomien und Konflikten (vgl. Abschnitt 2.1.2). Mit der Etablierung von Erziehungswissenschaft und Pädagogik als wissenschaftlichen Disziplinen veränderte sich auch die Ausbildung von Lehrern und Lehrerinnen. Die bis dahin unbestrittene Dominanz der ,pädagogischen Meisterlehre', wie sie in ihrer modernen Form in den Konzepten zur field-based teacher education stark verhaftet ist, trat zunehmend zugunsten der Betonung wissenschafticher Wissensrepertoires als Grundlage der Handlungssteuerung in Schule und Unterricht in den Hintergrund. Damit gewann allerdings auch die kritische Diskussion um die Handlungsferne pädagogischer Theoriebildung für das alltägliche Agieren in Schule und Unterricht an Gewicht. Seit Mitte der 1970er Jahre verstärkt sich in Deutschland die Bedeutung der so genannten empirischen Wende in der deutschen Erziehungswissenschaft; diese führt zu einer deutlichen Betonung von empirischer Lehr-Lern- und Bildungsforschung (als frühe Beispiele für Lehr-LernForschung vgl. z. B. die Beiträge in Treiber \& Weinert 1982); die dortigen Konzepte lassen sich gut in die derzeitigen Überlegungen zu evidenz-basierter Bildungspolitik integrieren (vgl. Abschnitt 2.2.1). Dieser Trend verstärkte die Distanz gegenüber Konzepten der pädagogischen Meisterlehre nochmals deutlich. Allerdings scheint seit einigen Jahren eine Annäherung dieser Positionen gegenüber solchen stattzufinden, in denen erfahrungsgestütztes Regelwissen dominiert. Diese Annäherung vollzieht sich u. a. im Rahmen der Debatte zum Novizen-Experten-Paradigma (z.B. Krauss 2011) sowie im Kontext der wissenspsychologischen Basierung professionellen Lehrhandelns (vgl. z. B. Voss, Kunter, Seiz, Hoehne \& Baumert 2014). So kann man die im Abschnitt 3.3.1 skizzierte Ausweitung der schulpraktischen Studienanteile als eine institutionelle Konsequenz dieses Diskussionstrends verstehen. Bedeutsam ist: Dies erfolgt in einer relativ engen Verknüpfung von Bildungspolitik mit Akteuren aus der empirischen Bildungsforschung (als letztes Beispiel vgl. das Gutachten zur Neustrukturierung der Berliner Lehrerbildung; Senatsverwaltung für Bildung, Jugend und Wissenschaft 2012).

Zur These der ,zerbrochenen 'Einheit von Theorie und Praxis: Vor dem oben skizzierten Hintergrund ist es nur folgerichtig, wenn Hedtke (2000: 1) formuliert: "Die Einheit von Theorie und Praxis, von Hochschule und Schule, Ausbildung und Beruf, ist verloren - wenn es sie denn je gegeben hat. Theorie und Praxis sind getrennt" (vgl. auch Radtke 2004: 111). Denn Erziehungswissenschaft auf der einen Seite und Erziehung auf der anderen Seite, hier Erziehung und Bildung in Schule, haben - so die Autorin - jeweils ein hohes Maß an eigener operativer Geschlossenheit entwickelt. Vor allem die ,Praxis' in Schule und Unterricht gehe mit einem deutlich erkennbaren Anspruch bezüglich der ihr eigenen kritischen Dignität an die ,Theorie' heran; d. h. die dortigen Akteure bewerten die Wissensrepertories, die Wissenschaft generiert und als zentrale Basis für professionelles Handeln deklariert, vor allem unter der folgenden Perspektive: Inwiefern dienen diese Wissensbestände der (reflexiven) Problembewältigung, vor Ort' bzw. inwiefern können sie dazu dienen? Deshalb hält es Hedtke (2000: 2) für unerlässlich, gerade den ,Praxis'bezug während der universitären Lehrerausbildung hinsichtlich seiner Formen und Funktionen konzeptionell neu zu verorten; sie hält es für höchst dysfunktional, diesen Bezug in handlungspraktisch gefassten feldbasierten Konzepten aufzulösen (auch Hedtke 2007). Folgt man diesen Überlegungen, erhält der Praxisschock im berufsbiographischen Prozesse der werdenden Lehrkraft eine konstruktiv-,aufschreckende' Funktion; denn er zeigt ihr die Begrenztheit der unterschiedlichen und mehrheitlich institutionell gebundenen Wissensrepertoires.

\subsubsection{Zum berufsbezogenen Wissen des lehrenden Akteurs}

In der deutschen Debatte zur Professionalität von Lehrkräften wird aktuell besonders die Bedeutung der Wissensrepertoires betont, die zur Handlungssteuerung verwendet werden. Dabei geht es primär um die Rolle der kognitiven Kontrolle des professionellen Handelns mittels dieser Repertoires (z. B. Reinisch 2009: 37ff.; auch Besser \& Krauss 2009: 78f.). „Wissen und Können“ werden als Kernbereich professioneller Kompetenz verstanden (vgl. z. B. Baumert \& Kunter 2011: 33ff.). In diesem Rahmen wird dem pädagogischpsychologischem Wissen eine deutlich stärkere Bedeutung beigemessen als bisher (vgl. z. B. Voss, Kunter, Seiz, Hoehne \& Baumert 2014). Gleichzeitig ist festzustellen, dass Emotion und Volition in diesen 
Die ,neue' Lehrerbildung in Deutschland. Vom Praxisschock zur aufgeklärten Aneignung professionellen Handelns?...

Konzepten nur eine Randrolle spielen. Beinah uni solo verweisen die meisten Autoren der aktuellen, empirisch ausgerichteten Debatte zu Professionalität und Professionalisierung auf Folgendes: Weitestgehend durchgesetzt hätten sich die Grundvorstellungen von Shulman (1986; 1987). Dieser Autor differenziert analytisch die folgenden Wissensarten - general pedagogical knowledge, subject matter content knowledge, pedagogical content knowledge, curricular knowledge, knowledge of learners und knowledge of educational context. Auch wenn diese Kategorisierung empirisch-analytisch nicht hinreichend valide belegt werden kann (vgl. z. B. Blömeke 2006; Voss, Kunter, Seiz, Hoehne \& Baumert 2014), so kommt ihr in Deutschland bildungspolitisch derzeit doch eine dominante Wirksamkeit zu; denn sie fungiert im Sinne auch eines konstruktiv-normativen Ordnungskonzepts für die Gestaltung von Curricula für die Ausbildung von Lehrerkräften. So bildet sie z. B. eine wesentliche Grundlage für die Beschlüsse der KMK (2004; 2010). Dabei zeichnet sich zwar durchaus ein Trend in Richtung field-based teacher education ab (vgl. z. B. Wisemann, Knight \& Cooner 2004; Zeichner 2010). Gleichwohl bleibt für die deutsche Diskussion das wissenschaftlich basierte Wissen auch für die berufswissenschaftlichen Studienanteile ein Eckpfeiler der curricularen Konstruktion. Gleichzeitig wird aber auch sichtbar, dass die Bedeutung epistemologischer Überzeugungen für die Generierung von Lehrprofessionalität zumindest bisher unterschätzt wird, wenn nicht weitestgehend ausgeblendet bleibt (zu diesen Überzeugungen vgl. z. B. Zinn 2013; vgl. Kitchener 1983 und Chinn \& Brewer 1993 zur Bedeutung dieser Überzeugungen in konfligierenden Situationen).

\subsection{Schulsystem, Schule und Unterricht - zum Neuen Steuerungssystem und zu systematischer Qualitätsentwicklung}

Neben den eher grundsätzlichen Überlegungen zu ,Theorie‘ und ,Praxis' und deren Wirkung auf die berufsbezogenen Wissensrepertoires, über die Lehrkräfte verfügen bzw. verfügen sollten, spielen Veränderungen in der Steuerung des Schulsystems sowie in der Qualitätsentwicklung der Einzelschule eine bedeutsame Rolle für die Gestaltung der strukturellen und inhaltlichen Reform der Lehrerbildung.

\subsubsection{Zur Outputorientierung von Schule und Unterricht im Rahmen evidenzbasierter Bildungspolitik}

Bildungspolitisch induziert, wird hinsichtich der Steuerung des Schulsystems seit ca. 15 Jahren ein gezielter Wechsel implementiert - von input-orientierter zu einer signifikant verstärkten output-orientierten Steuerung (vgl. z. B. Berkemeyer 2010: 24ff). Die Verstärkung der Output-Orientierung zeigt sich besonders in dem folgenden Umstand: Bei der Bewertung des Schulsystems einerseits sowie der Einzelschule andererseits wird den über die Schulcurricula definierten Lernergebnissen der Schüler und Schülerinnen ein zentrales Gewicht beigemessen (vgl. z. B. Köller 2007). Damit wird nicht zuletzt auch das Ziel verfolgt, zum Zwecke eines regelmäßigen Bildungsmonitorings zuverlässige empirische Befunde zum Zustand des Lernoutputs in dem einzelnen Bundesland zu generieren. In diese Perspektive eingeschlossen sind auch Outputvergleiche der 16 Bundesländer, zunehmend auch Vergleiche von Regionen innerhalb eines Bundeslandes bis hin zum Vergleich von Einzelschulen (vgl. z. B. Döbert \& Dedering 2008). Dieses Vorgehen fügt sich in die Konzepte evidenzbasierten politischen Entscheidungshandelns ein; diese werden seit ca. zwei Dekaden nicht nur für das Bildungssystem favorisiert, sondern haben z. B. auch das Gesundheitswesen erfasst (zu letzterem vgl. Lauterbach \& Schrappe 2001: 60ff.). Der vorrangige Anspruch ist, über den systematischen Bezug auf empirische Befunde eine höhere Rationalität des politischen Handelns sicherzustellen. Gleichzeitig mit diesen Veränderungen erfolgte die Integration von Konzepten des New Public Managements (kritisch dazu z. B. Wittmann 2007) in die Steuerungsstruktur, die bis dahin relativ einseitig durch top-down-Steuerungsstrategien gekennzeichnet war (vgl. z. B. Zlatkin-Troitschanskaia 2007: 71ff.). Deren ,Aufweichung' erfolgte zugunsten der Implementierung einer verstärkten Selbstständigkeit der Einzelschulen (dazu vgl. z. B. Rürup 2007). Durchaus im Sinne einer Gegenbewegung kann man inzwischen allerdings erkennen, dass Schritt für Schritt eine Verstärkung der externen Evaluation über stark standardisierte Instrumente vorgenommen wird. Eine Folge ist, dass der Raum für die Entwicklung einzelschulischer Qualitätsprofile begrenzt wird. Praktisch bundesweit eingesetzt werden Instrumente wie einzelschulübergreifende standardisierte Beurteilungsbögen für die einzelne Lehrkraft, Schulinspektion etc. (zur Schulinspektion vgl. z. B. die Beiträge in Müller, Pietsch \& Bos 2011; van Buer, Wagner, Rückmann \& Füssel 2015). Insgesamt ist zu erkennen: Das aus der Vergangenheit bereits bekannte Spannungsverhältnis zwischen zentraler Steuerung und einzelschulischem 
Entwicklungsbedürfnis baut sich erneut auf, derzeit allerdings im Rahmen einer veränderten Steuerungsstruktur. Zumindest von ihrem Anspruch her folgt das favorisierte Neue Steuerungsmodell (vgl. z. B. Altrichter \& Brüsemeister 2012) stärker Vorstellungen von Governance und Governing. Letztere werden im Wesentlichen verstanden als die stärkere Betonung der Qualität der Koordinations-, Kooperations- und Aushandlungsprozesse, die sich besonders an den inter- und intrainstitutionellen Schnittstellen ergeben (zur Educational Governance vgl. z. B. Heinrich 2012: 39ff.). Zentrales Ziel ist es, die dem Schulsystem zur Verfügung stehenden knappen Ressourcen zugunsten der Steigerung des Lernoutputs von Schule besser als bisher zu nutzen.

2.2.2. Zur Qualiätsentwicklung der Einzelschule in der Spannung zwischen lernender Organisation und ökonomischem Effektivitäts- und Effizienzkalkül

Die einschlägige Literatur zur Qualitätsentwicklung der Einzelschule ist kaum noch zu überblicken, weder international, noch für Deutschland. Sie überspannt ein breites Spektrum; dieses reicht von theoretischen Überlegungen zum ,Typus' der Institution Schule (zur Schultheorie vgl. z. B. Bohl, Harant \& Wacker 2014; Fend 2009), von Konzepten zu Schule als "lernender Organisation“ (für die deutsche Diskussion vgl. z. B. Schratz \& Steiner-Löffler 1999; Holtappels 2010), über die Diskussion zur Pädagogischen Schulentwicklung (vgl. z. B. Klippert 2000), weiter über die Debatte zu Führung und Qualitätsmanagement in Schule (vgl. z. B. Wagner 2011), über empirische Studien zu Einzelphänomenen von Schulentwicklung (vgl. z. B. Altrichter \& Helm 2011; Bohl, Helsper, Holtappels \& Schelle 2010) bis hin zu Handbüchern für die Führungs- und pädagogischen Akteure ,vor Ort' (vgl. z. B. Rolff 2011; Köller \& Pingel 2015). Darüber hinaus kann auf die Übersichtsartikel verwiesen werden, in denen versucht wird, über nationale Besonderheiten des Bildungssystems, von Schule und unterrichtlichen Traditionen hinaus allgemeine Merkmale zu identifizieren (vgl. z. B. Hattie 2009; Köller, Möller \& Möller 2013). Ohne im Rahmen dieses Beitrages auf Einzelheiten eingehen zu können, so kann man auch in internationaler Perspektive als zentrale Befunde festhalten: Die nachhaltige Entwicklung der Einzelschule ist ein Prozess, an dem willentlich und/oder unwillentlich alle Akteure der jeweiligen Institution sowie Außenstehende wie Eltern, lokale und regionale Unternehmen etc. beteiligt sind (zu innerschulischen Lehrergruppierungen vgl. z. B. Köller 2009); gleichzeitig ist Schulentwicklung ebenfalls ein Prozess, der insgesamt brüchig verläuft (vgl. z. B. Baum 2014: 183ff.) und dessen Ergebnisse letztlich immer nur als Zwischenergebnisse auf einem unsicheren Entwicklungsweg verbleiben (vgl. Z. B. Rolff 2014). In den einschlägigen Konzepten bildet sich mehrheitlich der Kreislauf von „Plan - Do - Check - Act“ ab, auf den bereits Deming (1982) im Rahmen ökonomisch orientierter Qualitätskonzepte aufmerksam gemacht hat; dieser Qualitätszyklus bildet inzwischen die mehr oder weniger explizite Grundvorstellung für die Gestaltung nachhaltiger Schulentwicklung (vgl. z. B. die Beiträge in Arnold \& Prescher 2014).

Gerade die letzteren Konzepte führen zu einer Verknüpfung von einzelschulinterner Entwicklungsanstrengung mit solchen Formen von Schulsystementwicklung, die sich in den Rahmen von New Public Management, Neuem Steuerungsmodell und evidenzbasierter Schulpolitik einordnen lassen. Generell geht es in dem oben angesprochenen Deming-Kreislauf von Qualitätsentwicklung darum, die Unterrichtsprozesse und die einzelschulische Ressourcenverwendung zu optimieren; dabei sollte so weit wie möglich datenbasiert vorgegangen werden. Ziel ist, dadurch die schrittweise Verbesserung des Lernoutputs in der Einzelschule zu erreichen (vgl. z. B. Lehmann, Peek, Gänsfuß, Lutkat, Mücke \& Barth 2000). Einen weiteren Hintergrund für diese Vorstellungen stellt das Bildungsproduktionsmodell (vgl. z. B. Timmermann \& Windschild 1996) dar. Ursprünglich ist dieses für die betriebliche Aus- und Weiterbildung entwickelt; es zielt daher besonders auf die Kundenzufriedenheit sowie auf den Transfer des erworbenen Wissens in den betrieblichen Arbeitszusammenhang. Gleichwohl hat es Eingang gefunden in die deutsche Debatte zur Qualitätsentwicklung von Schule; denn es beruht auf ähnlichen kybernetisch ausgerichteten „Annahmen des Zusammenhangs von Input, Prozess und Output" (Berkemeyer 2010: 54) wie der für Schulentwicklung adaptierte Deming-Zyklus. Eines der Merkmale, das als Vorteil des Bildungsproduktionsmodells benannt wird, ist die Ausrichtung des Modells auf die Rechenschaftslegung für alle Prozess- und Ressourcenkomponenten der einzelnen Bildungsinstitution; letztlich führt dies zur Orientierung des Entscheidungshandelns an der quantitativ ermittelten (Bildungs-)Rendite. Für Schule bedeutet dies in der Regel den Versuch, eine quantifizierende Einschätzung des Verhältnisses von Inputleistungen (Kosten für die Lehrkräfte, laufende Kosten etc.) und erreichtem Lernoutputs der Schüler zu 
Die ,neue' Lehrerbildung in Deutschland. Vom Praxisschock zur aufgeklärten Aneignung professionellen Handelns?...

erreichen. Allerdings sieht sich das Bildungsproduktionsmodell starker erziehungswissenschaftlichpädagogischer Kritik ausgesetzt; diese verweist auf die relativ strikte Ausrichtung des einzelschulischen Gesamtprozesses auf ein ökonomisch basiertes Effektivitäts- und Effizienzkonzept bzw. -kalkül (vgl. z. B. Heid 2007). Insgesamt stellt sich durchaus die kritische Frage, inwiefern das weithin favorisierte Verständnis von Schule als "lernender Organisation“ (vgl. z. B. Holtappels 2010) einen wirksamen Gegenpol oder letztlich nur eine Moderierung der weiter oben skizzierten Trends hin zu einem ökonomisch ausgerichteten Effektivitäts- und Effizienzverständnis darstellt. Abschließend beantwortbar ist diese Frage derzeit für Deutschland nicht. Dabei spielt sich diese Steuerungsfrage in Deutschland zunehmend eher im Hintergrund ab; denn Kernpunkte der aktuellen bildungspolitischen Diskussion werden derzeit durch Forderungen besetzt, inklusiven Unterricht signifikant stärker als bisher zu implementieren (vgl. z. B. KMK 2015; kritisch Ahrbeck 2014) sowie die stärkere Integration von Migranten und Migrantinnen in Schule und Unterricht zu verwirklichen (vgl. z. B. KMK 2013a; Ceri 2015). Allerdings verschärft sich damit auch die Frage nach angemessener Ausfinanzierung von Schule und Unterricht (vgl. z. B. Klemm \& Preuss-Lausitz 2011: 111ff.). Quasi durch die Hintertür wächst die Wahrscheinlichkeit, bei knappen Ressourcen der öffentlichen Hand ökonomisch begründete Effektivitäts- und Effizienzvorstellungen durchzusetzen (zu Finanzierungsproblemen von Schule vgl. z. B. Eisinger, Warndorf, Falterbaum \& Feldt 2010). Eine Konsequenz aus den oben skizzierten Trends ist die Diskussion, Schulen zunehmend als Bildungsdienstleister zu verstehen, die sich untereinander im Wettbewerb um Bildungskunden befinden (vgl. z. B. Altrichter, Heinrich \& SoukupAltrichter 2011). Vor allem bei zurückgehenden Schülerzahlen in den meisten Regionen Deutschlands haben sie - so die Konsequenz - ihr längerfristiges Überleben über besondere Leistungen etc. zu sichern, die sie mittels von Marketingstrategien auf dem Bildungsmarkt anbieten (zu Bildungsmarketing vgl. z. B. Barz 2010: 415ff.). Auf diese stark ökonomisierende Perspektive für Unterricht, die nicht zuletzt über Bildungspolitik und Schuladministration in die Arbeit ,vor Ort', durchgereicht' wird, reagieren Lehrkräfte in Deutschland mit vergleichsweise starker Ablehnung. Denn ihre berufliche Identität ist primär an einem Bild inrer Tätigkeitsstruktur orientiert, das in besonderem Maße die drei folgenden Kernaspekte beinhaltet: (a) die Verpflichtung des Staates zur Grundfinanzierung der öffentlichen Schulen, (b) damit auch die Nichtorientierung an ökonomisch ausgerichteten Kalkülen der innerschulischen Ressourcenverwendung und (c) die pädagogische Autonomie der einzelnen Lehrkraft als verbürgtes Recht ihrer Profession (vgl. z. B. van Buer 1990; Fauser 1986). Letzteres schließt nicht zuletzt auch die Autonomie gerade gegenüber zu strikter Ausrichtung der pädagogischen Arbeit in Schule und Unterricht auf die Optionen des Arbeitsmarktes ein.

\subsection{Reform der Lehrerbildung als systemischer Bestandteil des Gesamtkonzepts der Erneuerung des deutschen Schulwesens}

Gerade angesichts des anstehenden massiven Wechsels des Lehrpersonals an den Schulen (vgl. Abschnitt 1) kommt der Berufsausbildung der neuen Lehrkräfte eine besondere Rolle für die Initiierung und für Verstetigung veränderter Schul- und Unterrichtskultur zu. Denn die Vorstellungen über Schule, mit denen die derzeitigen Studierenden des Lehramtes aufgewachsen sind und die sie über ihre Berufsentscheidung mit in ihr Studium nehmen, erscheinen hoch stabil (vgl. Kuhlee, van Buer \& Klinke 2009:151ff.). Gleichzeitig ist erwartbar: Angesichts der oben skizzierten signifikanten Veränderungen in Schule und in Unterricht bilden diese subjektiven Vorstellungen der Studieneinsteiger nur noch bedingt die Strukturen und Anforderungen ab, auf die Berufseinsteiger dann ca. 6 Jahre später treffen werden (vgl. Kuhlee \& van Buer 2009).

Für die Lehrerbildung stellen sich damit Fragen nach einer weitgehenden curricularen Reform der Studienangebote, aber auch nach einer tiefergreifenden strukturellen Veränderung der Studiengänge. In den letzten ca. 15 Jahren hat die Kultusministerkonferenz (KMK = Konferenz der für das Schulsystem zuständigen Kultusminister der 16 Bundesländer) Schritt für Schritt den Anspruch verstärkt, die von ihr formulierten Änderungsperspektiven (vgl. z. B. KMK 2001; 2007; 2013a) sowie die Rahmenprogramme zur Schul-, Unterrichts- und Fachentwicklung (als Beispiel vgl. KMK 2009a; 2009b; 2011b) als Teile eines systemisch integrierten Gesamtprogramms der grundlegenden Reform des Schulwesens, der Einzelschule und der Unterrichtskultur zu konstruieren. Für Deutschland neu ist, die Berufsausbildung der späteren Lehrkräfte von Anfang an in das Netzwerk von Reformperspektive, Rahmenprogramm und Instrumenten systematisch einzubeziehen. Damit verknüpft ist der Anspruch, stärker als in der Vergangenheit steuernd in die universitären Studienangebote einzugreifen (vgl. z. B. HRK 2006; kritisch vgl. z. B. HRK 2011). 


\section{Akademische Berufsausbildung von Lehrerkräften in Deutschland - zu strukturellen Kontinuitäten und curricularen Veränderungen}

„Neue Lehrer braucht das Land", so beginnt der Abschnitt 1 mit seinem Verweis auf die hohe Aktualität der Debatten um die Restrukturierung der Lehrerbildung. In der öffentlichen wie auch in der bildungspolitischen Diskussion werden als zentrale Kontexte für diese Forderungen die Veränderungen in Schule und Unterricht angeführt. Über die Frage nach dem Verhältnis von ,Theorie' und ,Praxis' wird dabei öffentlich eher beiläufig gesprochen. Insgesamt erscheint es in der bildungspolitischen Debatte jedoch beinah als selbstverständlich, bereits in der Phase ihres universitären Studiums signifikant stärker als bisher die ,Praxisphasen', also der Besuch der feldbasierten Erfahrungsräume in Schule und Unterricht, in der Berufsausbildung der künftigen Lehrkräfte auszuweiten. Häufig als „Praxisschock“ bezeichnet, soll damit dem von den Studierenden häufig als konfliktreich erlebten Übergang von der Phase ihres universitären Studiums in die Phase des staatlichen Vorbereitungsdienstes vorgebeugt werden.

\subsection{Allgemeine Rahmenbedingungen für die deutsche Lehrerbildung}

In vielen einschlägigen Publikationen zur deutschen Lehrerbildung bleiben die rechtlichen Rahmenbedingungen unbeachtet bzw. werden in ihren Effekten unterschätzt. Gerade im Vergleich mit anderen Ländern erweisen sie sich jedoch als bedeutsam für das Verständnis der deutschen Lehrerbildung (vgl. z. B. die Beiträge in Kuhlee, van Buer \& Winch 2015 zum Vergleich der deutschen und englischen Lehrerbildung). Wie in den Abschnitten 2.2.1 und 2.2.2 skizziert, vollzieht sich die Restrukturierung vor allem der universitären Phase der Lehrerbildung in hohem Maße in systemischer Vernetzung mit den Veränderungen in der Steuerung des Schulwesens sowie in Schule und Unterricht. Die Frage, in welchem Ausmaß der Staat auf Veränderungen in der universitären Lehrerbildung in Deutschland drängt bzw. zu drängen in der Lage ist, ist wesentlich durch fünf Aspekte definiert. Diese sind historisch gewachsen; gleichzeitig stellen sie jedoch auch aktuell wirksame gesellschaftliche Leitbilder dar.

(1) Hoheit des Staates über das Schulwesen: Im Artikel 7, Absatz 1, des Grundgesetzes (deutsche Verfassung) ist festgelegt: „Das gesamte Schulwesen steht unter Aufsicht des Staates“. Zwar wird die Errichtung privater Schulen im Absatz 4 desselben Artikels gewährleistet; jedoch bedürfen diese der "Genehmigung des Staates und unterstehen den Landesgesetzen". Insgesamt ergibt sich daraus auch das Verständnis, dem Staat - hier den Bundesländern - obliege es, den Zugang zum Arbeitsmarkt von Lehrkräften an öffentlichen Schulen zu kontrollieren. Dies wird dadurch realisiert, dass mit Ausnahme der so genannten Mangelfächer wie Physik, Chemie, Mathematik, Informationstechnik (KMK 2011a) und teilweise auch für die Lehrkräfte an beruflichen Schulen der Zugang zu diesem Marktsegment ausschließlich über die zweistufige Berufsausbildung zum Lehrer bzw. zur Lehrerin geöffnet ist (deutlich anders z. B. in England; vgl. Sprigade 2015).

(2) Föderalistische Grundstruktur des deutschen Bildungswesens: Für das deutsche Schulwesen gilt die föderalistische Grundstruktur der Bundesrepublik (vgl. z. B. Rürup 2005). In der Folge sind die 16 Bundesländer der Bundesrepublik für ihr Schulwesen eigenständig verantwortlich und können dieses nach eigenem Ermessen entwickeln. Um die Disparitäten zwischen den Bundesländern und damit den Bildungsregionalismus überschaubar zu halten, wurde das Instrument der Ständigen Konferenz der Kultusminister der Länder (KMK) geschaffen. Mit diesem Steuerungsinstrument versuchen die Kultusminister, einen für alle Bundesländer tragfähigen Konsens z. B. bezüglich der Strukturierung des Schulwesens, der curricularen Entwicklung der Schulfächer, aber auch hinsichtlich der Strukturierung und curricularen Profilbildung der Lehrerbildung zu generieren (vgl. KMK 2004). Gleichwohl sind im Vergleich der Bundesländer gerade hinsichtlich der Binnenstruktur der Lehrerbildung nach wie vor signifikante Unterschiede erkennbar (vgl. Abschnitt 3.3 zu den ,Praxisphasen' in der Lehrerbildung).

(3) Lehrkräfte als Personen mit hoheitlichen Befugnissen: Vor allem in den westdeutschen Bundesländern werden die Lehrkräfte der öffentlichen Schulen als Beamte (tenured civil servants) eingestellt (vgl. z. B. Füssel \& van Buer 2015). Grundlage dafür sind vor allem verfassungsrechtliche Überlegungen; diese verweisen auf Folgendes: „Die Ausübung hoheitsrechtlicher Befugnisse ist als ständige Aufgabe in der Regel Angehörigen des öffentlichen Dienstes zu übertragen, die in einem öffentlich- 
Die ,neue' Lehrerbildung in Deutschland. Vom Praxisschock zur aufgeklärten Aneignung professionellen Handelns?...

rechtlichen Dienst- und Treueverhältnis stehen" (Füssel \& van Buer 2015: 136). Damit wird die Tätigkeit einer Lehrkraft in einer öffentlichen Schule als „Aufgabe hoheitsrechtlicher Natur" verstanden; letztere wirkt „durch Befehl oder Zwang unmittelbar beschränkend auf grundrechtlich geschützte Freiheiten" (138) ein. In der Folge kommt dem Staat eine besondere Verpflichtung in der Steuerung und Kontrolle des in sich weitgehend geschlossenen Lehrerarbeitsmarktes zu; dies gilt in besonderem Maße für den Zugang zu diesem Marksegment (vgl. Punkt 1).

(4) Zweistufige Berufsausbildung der Lehrkräfte an öffentlichen Schulen: Auffällig und gegenüber den meisten anderen europäischen Staaten unterschiedlich ist die phasenmäßige Organisation der deutschen Lehrerbildung; sie wird von den Studierenden in zwei Stufen durchlaufen. Sie beginnen ihre Ausbildung mit der Stufe des Lehramtsstudiums an einer Hochschule. Danach gehen sie in die Stufe des Vorbereitungsdienstes über (Referendariat); diese Stufe liegt vollständig in staatlicher Hand. Auf der einen Seite beziehen sich die Begründungen für diese Struktur auf die in den Punkten (1)-(3) skizzierten Argumente; auf der anderen Seite sind die Ähnlichkeiten zum korporatistischen System der nichtakademischen Berufsausbildung, dem so genannten Dualen System, unübersehbar (dazu vgl. z. B. Stender 2011).

(5) Autonomie der Hochschule: Im Artikel 5, Absatz 3, des Grundgesetzes wird formuliert: „Kunst und Wissenschaft, Forschung und Lehre sind frei". Dabei werden Freiheit in Wissenschaft, Forschung und Lehre nach gängiger Rechtsauffassung als ein eng verknüpftes einheitliches Grundrecht verstanden. Zudem sei Forschungsfreiheit als vorbehaltloses Grundrecht zu gewährleisten. Folgt man z. B. Lorenz (1981), ist die Rechtsstellung der Universität als „Autonomie im Staat” zu verstehen (vgl. Rogal 2008: 18ff.). Hinsichtlich der ersten Phase der Lehrerbildung an einer Hochschule stellt sich damit die Frage, wie weit das jeweilige Bundesland normierend eingreifen und damit die Autonomie der Hochschule zumindest bedrängen darf.

\subsection{Grundstruktur der deutschen Lehrerbildung}

Im Jahr 2004 folgend wurde die deutsche Lehrerbildung umgestellt - vom 8-/9semestrigen Modell des 1. Staatsexamens (Lehrkräfte der Primarstufe $=6$ Semester) und des 2 . Staatsexamens zum Abschluss des staatlichen Vorbereitungsdienstes (Referendariat; je nach Schulstufe 1 - 2 Jahre) auf die BachelorMaster-Struktur. Insgesamt haben sich die sechszehn Bundesländer darauf geeinigt, bei der Umstellung auf diese neue Struktur einen verbindlichen Strukturrahmen zu verabschieden. Dieser solle zur bundesweiten Anerkennung der Studienabschlüsse im Lehramt führen (vgl. KMK 2008; 2013b; zur Übersicht über die Bundesländer vgl. KMK 2014). Dieser Strukturrahmen lässt jedem Bundesland jedoch hinreichenden Raum, in Absprache mit seinen Hochschulen seine eigenen Vorstellungen zu implementieren. Dabei betreffen diese vor allem die curricularen Binnenstrukturen der Studienprogramme, nicht zuletzt auch den Umfang der Schulpraktika und damit das Verhältnis von theorieorientiertem und erfahrungsgebundenem Lernen (als Überblick vgl. Hilligus 2015; Kuhlee 2015). Mit dem Beschluss der KMK (2004) werden wesentliche und damit verbindliche Strukturmerkmale für die deutsche Lehrerbildung festgelegt. Zum einen befinden sich diese in der Kontinuität des, alten' Staatsexamensmodells (vgl. unten Merkmale 1 - 6); zum anderen werden aber auch Änderungen sichtbar (Merkmale 7 - 13) (zum internationalen Vergleich vgl. z. B. Blömeke 2006: 395ff.).

Die unten skizzierten Gemeinsamkeiten der Reformen bzw. der Kontuinierung bisheriger Merkmale beziehen sich primär auf die strukturelle Ausrichtung des Lehramtsstudiums. Hinsichtlich der curricularen Binnenstrukturen ist es der KMK zwar ebenfalls gelungen, Standards auszuhandeln (vgl. KMK 2004). Diese sind jedoch deutlich ,vager' im Sinne von Leitlinien formuliert. Ein wichtiger Grund dafür, jedem Bundesland ein erhebliches Maß an curricularen Freiräumen sowohl in den fachwissenschaftlichen Studienanteilen als auch in den Studienanteilen der Bildungswissenschaften zu belassen, ist in dem folgenden Umstand zu sehen: Viele Bundesländer verfügen über ihren eigenen Qualitätsrahmen, den sie für die Steuerung des Schulsystems generiert haben; diesen wollen sie auch als Rahmendefinition für die curriculare Ausrichtung der bildungswissenschaftlichen Anteile des Lehramtsstudiums verwenden (für das Bundesland Berlin vgl. van Buer, Kohring \& Frasch 2009, 40ff.). Ein weiterer Grund ist in der Hochschulautonomie der ausbildenden Hochschulen und Universitäten zu sehen (vgl. Abschnitt 3.1, Punkt 5). 
Als generelle curriculare Leitlinie kann festgehalten werden: Als entscheidende Wissensqualität und vor allem auch als prüfungsrelevante Wissensrepertoires für die universitäre Lehrerbildung gelten (a) theoriebasiertes Wissen sowohl in den fachwissenschaftlichen Studienanteilen der beiden zu studierenden Fächer (z. B. in Biologie und Sport) als auch (b) in Erziehungswissenschaften sowie in jeder der beiden zuständigen Fachdidaktiken. In der zweiten Stufe der Ausbildung, im staatlichen Vorbereitungsdienst (Referendariat), spielen die Moderierung, Anpassung an die alltäglichen handlungspraktischen Bedürfnisse der Referendare und gegebenenfalls auch das Außer-Kraft-Setzen des universitär erworbenen Wissens eine bedeutsame Rolle. Zu erwarten ist: Die Bedeutung theoriebasierten Wissens wird in dieser zweiten Phase signifikant durch regulatives, erfahrungsgestütztes und situationsbezogenes Wissen relativiert. Wie z. B. Blömeke (2006) in ihrer internationalen Vergleichsstudie feststellt, führt diese Organisation von Studien- und Lernangeboten über die Zeit hinweg zu einer eklatanten Fragmentierung des insgesamt zu erlernenden Wissens.

Sechs Kontinuitätsmerkmale von ,alter' in die ,neue 'Lehrerbildung: (1) Die schulartbezogene Ausbildung wird fortgesetzt bzw. weiter verstärkt. (2) Es sind weiterhin zwei Fächer zu studieren (z. B. Deutsch und Englisch). Die zugelassenen Fächer und deren Kombinierbarkeit sind in den so genannten "Landeslisten" festgelegt. Dabei gelten die in diesen Listen zugelassenen Fächer bundesweit; allerdings weist deren Kombinierbarkeit im Vergleich der Bundesländer Unterschiede auf, die wesentlich auf die bundeslandspezifische Bedarfssituation zurückzuführen sind. (3) Der relative Studienanteil des Erst- und des Zweitfaches (jetzt Kernfach und Zweitfach genannt) wird im Wesentlichen erhalten. Der Gewinn an Studienzeit, der durch das im Bachelor-Master-Modell um 1-2 Semester längere Studium erzielt wird, kommt primär dem Studienangebot des Zweitfaches sowie der Berufswissenschaften zugute. (4) Die Kombination von Erziehungswissenschaften, Fachdidaktik für das 1. Fach und Fachdidaktik für das 2. Fach bleibt erhalten. Dabei nimmt dieser Studienanteil, jetzt unter dem Namen „Berufswissenschaften“ zusammengefasst, signifikant zu. (5) Im Grundgesetz, Artikel 12, ist das Grundrecht auf Berufsfreiheit festgelegt. In der Folge ist für die nicht-akademische wie auch für die akademische Berufsausbildung bindend: Jede Person, die eine Berufsausbildung begonnen hat, hat das Recht, diese nach Beendigung der Probezeit im Rahmen von Regelzeiten zu beendigen. In der Folge hat jeder Studierende bzw. jede Studierende, die das Studium des Lehramtes im Bachelor begonnen hat, das Recht, unabhängig von der im Bachelorstudium erzielten Abschlusszensur in den Master of Education zu wechseln. Des Weiteren hat er bzw. sie das Recht, nach erfolgreichem Masterabschluss in den staatlichen Vorbereitungsdienst überzugehen. Damit sind die Lehramtsstudiengänge zusammen mit nur wenigen anderen Bachelorstudiengängen die einzigen, für die keine Selektionsschranke zwischen den Studienstufen Bachelor - Master zulässig ist. (6) Die Berufsausbildung zur Lehrkraft für eine öffentliche Schule endet nach wie vor mit dem Staatsexamen. Dieses wird am Ende des staatlichen Vorbereitungsdienstes vor einer rein staatlichen Kommission abgelegt; an dieser sind Vertreter und Vertreterinnen des universitären Studienangebots nicht beteiligt.

Sieben Veränderungsmerkmale im Vergleich von ,alter' und ,neuer' Lehrerbildung (vgl. KMK 2014): (7) Die Lehramtsstudiengänge sind durch externe Akkreditierungsunternehmen zu bewerten. Erst durch die erfolgreiche Akkreditierung erlangt ein Studiengang des Lehramtes seine bundesweite Akzeptanz. (8) In jedem Bundesland werden an ausgewählten Hochschulen Lehrerbildungszentren geschaffen. Ihre Aufgabe ist es u. a., die Fragmentierung der Studieninhalte, hier besonders im Bereich der Berufswissenschaften, zu überwinden (zur Fragmentierung von Studieninhalten im Lehramtsstudium im internationalen Vergleich vgl. z. B. Blömeke 2006). Derzeit sind an 54 Hochschulen Zentren dieser Art eingerichtet (vgl. Monitor Lehrerbildung 2015). (9) Die Stufe des Bachelorstudiums im Lehramt umfasst eine Regelstudienzeit von 6 Semestern, die Stufe des Master of Education eine Regelstudienzeit von 4 Semestern. (10) Am Ende des Bachelorstudiums wird die wissenschaftliche Abschlussarbeit im Kernfach geschrieben, bezieht sich also auf das fachwissenschaftliche Studium. Die Abschlussarbeit im Master of Education kann in einem der beiden Fächer wie auch in den Berufswissenschaften geschrieben werden. (11) Die gesamte Studienstruktur wird modularisiert. Die Studienleistungen werden über Kreditpunkte (CP) berechnet; diese sind über Zeiteinheiten (derzeit $1 \mathrm{CP} \approx 25-30$ auf das Studium zu verwendende Stunden) ermittelt. (12) Mindestens 1 Schulpraktikum ist in das Studium zu integrieren. In der Regel ist dies das Unterrichtspraktikum, in dem der oder die Studierende in jedem der beiden studierten Fächer unter Anleitung einer erfahrenen Lehrkraft Unterricht erteilt (genauer vgl. Abschnitt 3.4). Dieses Praktikum wird in fast allen Hochschulen von einem dort Lehrenden aus der jeweiligen Fachdidaktik begleitet. (13) Nicht 
Die ,neue' Lehrerbildung in Deutschland. Vom Praxisschock zur aufgeklärten Aneignung professionellen Handelns?...

zuletzt aus pragmatischen Gründen der Finanzierbarkeit wird auch für den lehramtsbezogenen Bachelor die allgemeine Bachelor-Grundstruktur erhalten. Danach sind ca. $30 \mathrm{CP}$ dem Studium Generale als freiem Wahlbereich zuzuordnen. Im Lehramtsstudium entfällt allerdings die freie Wahl; denn dieser Bereich wird durch die Berufswissenschaften eingenommen, die in der Regel die gesamte Anzahl von CP mit obligatorischen Studieninhalten belegen.

\subsection{Umfang und Struktur des erfahrungsbasierten Lernens in Schule und Unterricht in der universitären Lehrerbildung}

\subsubsection{Praxisphasen in der deutschen Lehrerbildung - eine formal-quantitative Annäherung}

Wie im Abschnitt 3.2 beschrieben, zielt die Reform der universitären Lehrerbildung einerseits auf die inhaltliche Neugliederung der einzelnen Studienbereiche, dort vor allem in den Berufswissenschaften; andererseits führt diese Reform auch zu einer formal-zeitlichen Aufstockung der Anteile erfahrungsgebundenen Lernens vor Ort in Schule und Unterricht. Diese Aufstockung ist primär im Master of Education platziert, wo in den berufswissenschaftlichen Studienanteilen gezielt eine neue Ausbalancierung von theorieorientiertem und erfahrungsgebundenem Lernen angestrebt wird. In dieser Studienstufe werden die formal-strukturelle, die inhaltlich-curriculare und die empirisch basierte Beantwortung der Generalfrage dieses Beitrages - vom Praxisschock zur aufgeklärten Aneignung professionellen Handelns - am sichtbarsten. Zwar hat die KMK derzeit noch keine explizite gemeinsame Erklärung über Struktur und Inhalt des Praxissemesters verfasst; gleichwohl zeigt der Blick in das Internet unter dem Stichwort „Praxissemester": Die Mehrzahl der Hochschulen, in denen Lehrerbildung angeboten wird, weisen das Praxissemester als ein besonderes Profilmerkmal im Sinne eines signifikanten Entwicklungsprojektes aus. Angesichts der föderalistischen Grundstruktur der Bundesrepublik (vgl. Abschnitt 3.1) ist es nicht überraschend, dass gerade für die Neukonstruktion der Praxisphase(n) im Vergleich der Bundesländer massive Unterschiede festzustellen sind. Bezüglich der formalen und zeitlichen Struktur des ,Praxis'anteils am Studium können vor allem die folgenden vier Merkmale festgehalten werden:

(a) Ungenauer Überblick über die realen Zeitanteile erfahrungsbasierten Lernens in der universitären Lehrerbildung: Zur Frage, wie viel Studienzeit in den pädagogischen sowie fachdidaktischen Seminaren, Übungen etc. auf Simulationen von unterrichtlicher, Praxis' verwendet wird (z. B. in den Didaktiklaboren der Fachdidaktiken bzw. der Grundschulpädagogik), liegen keine zuverlässigen Informationen vor (vgl. z. B. Hascher 2012). Allerdings ist erwartbar, dass dieser Zeitanteil in den Bachelor- und Mastermodellen des Lehramts in den letzten Jahren gestiegen ist (z. B. die Beiträge in Roters, Schneider, Koch-Priewe, Thiele \& Wildt 2009). Präzisere Auskünfte sind bezüglich der rechtlich geregelten Studienzeitkontingente möglich, die im Rahmen von Praktika und/oder Praxissemester auf den Kontakt mit bzw. auf Lehren in Schule verwendet werden sollen. Diesbezüglich varieren diese Kontingente erheblich zwischen den Bundesländern. In den Flächenstaaten wie Nordrhein-Westfalen oder Hessen ist dies nochmals im Vergleich der ausbildenden Universitäten innerhalb des einzelnen Bundeslandes feststellbar (vgl. z. B. Bosse 2012: 4).

(b) Zum Zeitumfang der ,Praxis‘anteile: Eine genauere Übersicht pro Bundesland ist in der Zusammenstellung der KMK (2014) zu finden. Im Folgenden sind einige Beispiele angeführt; sie verdeutlichen die große Variationsbreite im Vergleich der Ausbildungsstandorte: Im Bundesland SachsenAnhalt sind auf die ,praktischen' Studienanteile 110 Stunden zu verwenden (Ausbildung für die Sekundarstufe II). Im Bundesland Berlin umfassen die drei obligatorischen Schulpraktika derzeit insgesamt 174 Stunden; ab dem Wintersemester 2016/17 wird das Praxissemester verbindlich für alle Studiengänge der Lehrerbildung eingeführt. In Niedersachsen umfasst der Praxisanteil 270 Stunden. In Brandenburg haben die Studierenden insgesamt 19 Wochen sowie zwei Mal ein semesterbegleitendes Tagespraktikum zu absolvieren. In Thüringen (an der Universität Jena) sind es 330 Stunden sowie 22 Wochen. Folgt man Offenberg \& Walke (2013: 106), finden gerade die kontroversen Diskussionen zur Ausweitung des Zeitanteils eher auf der organisatorisch-verfahrenstechnischen Ebene statt; dabei wird die Sinnhaftigkeit dieser Ausweitung nur selten in Frage gestellt.

(c) Zur Binnenstruktur des ,Praxisanteils: (c1) In Berlin durchlaufen die Studierenden aktuell ein Einstiegspraktikum auf der Bachelorstufe. Im Master of Education werden ab Wintersemester 2016/2017 die bisherigen zwei vierwöchigen Unterrichtspraktika mit fünftägiger Anwesenheit in der Schule (in der Regel 
während der vorlesungsfreien Zeit) durch ein Praxissemester ersetzt; in dieser Zeit sind die Studierenden drei Tage pro Woche in der Schule, visitieren Unterricht in ihren beiden Fächern und halten in jedem ihrer beiden Fächer je 18 Stunden zunehmend selbstständigen Unterricht. Planung, Durchführung und Evaluierung dieses Probeunterrichts werden von erfahrenen Lehrkräften sowie dem zuständigen universitären Dozenten der Fachdidaktik begleitet. Diese Praxisphase ist gekoppelt mit einer anwendungsorientierten Forschungsarbeit der Studierenden, in der sie individuell ein schul- bzw. unterrichtsrelevantes Problemfeld bearbeiten und ihre Überlegungen so weit wie möglich mittels einer kleinen empirischen Studie absichern. (c2) In Thüringen (Jenaer Modell) ist die Gesamtzeit, die für den Erwerb von ,Praxiserfahrungen' vorgesehen ist, aufgeteilt in ein Einstiegspraktikum und in das fachdidaktische Praktikum jeweils für die beiden fachwissenschaftlichen Studienfächer (für das Jenaer Modell; vgl. z. B. Jantowski 2010; die Beiträge in Kleinespel 2014). (c3) In Baden-Württemberg umfasst das Schulpraxissemester 13 Wochen als Blockpraktikum (Ministerium für Kultus, Jugend und Sport 2010). (c4) In Brandenburg ist diese Praktikumszeit nochmals differenzierter aufgebaut; sie ist aufgeteilt in ein Einstiegspraktikum, in ein pädagogisch-psychologisches Praktikum, in ein psychodiagnostisches Praktikum, in ein Praxissemester und in zwei semesterbegleitende Tagespraktika (zum Überblick über die Praxissemester Weyland \& Wittmann 2011: 12ff.).

(d) Zu institutionellen Unterstützungen: Die im Verlaufe der letzten Dekade eingeführten Bachelor- und Masterprogramme für das Lehramt werden durch zwei Entwicklungen unterstützt: Zum einen haben aktuell 54 Universitäten zur Lehrerbildung eigene Lehrerbildungszentren aufgebaut (vgl. Monitor Lehrerbildung 2015; vgl. KMK 2014; die Beiträge z. B. in Kraler \& Schratz 2008). Zum anderen haben Bundesregierung und Bundesländer massive Investitionen in die empirische Erforschung der Effekte dieser neuen Konzepte und deren nachhaltige Etablierung getätigt (vgl. Abschnitt 1).

\subsubsection{Praxisphasen in der deutschen Lehrerbildung - eine curricular-inhaltliche Annäherung}

Bezüglich der inhaltlich-konzeptionellen Grundlegung können für die deutsche Lehrerausbildung aktuell zwei durchaus widersprüchliche Trends festgestellt werden. Auf der einen Seite: In der einschlägigen Diskussion und auch in den (bildungs-)politischen Publikationen ist die generelle Betonung von kompetenzorientierter Ausbildung unübersehbar (vgl. z. B. Senatsverwaltung für Bildung, Jugend und Wissenschaft 2014: 21ff.). Die KMK (2004) hat diese Orientierung als generelle Leitlinie für die anstehenden Reformen beschlossen und nachfolgend im Sinne von Standards mehrfach bestätigt (vgl. KMK 2008; 2010); anschließend wurden diese in den Bundesländern spezifiziert. Diese Standards sind strukturell ähnlich den Bildungsstandards für das Schulwesen aufgebaut. Somit zeigt sich auch hier die im Abschnitt 2.3 herausgestellte bildungspolitische Intention, die Veränderung von Schulsystem, Einzelschule und Unterricht systemisch mit der Reform der Lehrerbildung zu vernetzen. Wie ebenfalls bereits mehrfach angesprochen, gilt dies in besonderem Maße für die berufswissenschaftlichen Inhalte der Studienprogramme. Im Vorbereitungsdienst, der sich in rein staatlicher Obhut befindet, sind diese Verknüpfungen nochmals stärker zu erkennen (für das Bundesland Berlin vgl. Senatsverwaltung für Bildung, Jugend und Wissenschaft 2014).

Bei der inhaltlichen Ausgestaltung der Studiengänge für das Lehramt verbleibt die Festlegung der fachwissenschaftlichen Studienanteile weitgehend in der Hand der einzelnen Universität. Für die Berufswissenschaften hingegen benennt die KMK (2004: 4f.) obligatorische Bestandteile der Ausbildung in Erziehungswissenschaft, Pädagogik und Fachdidaktik (vgl. auch Terhart 2006). Damit wirkt sie relativ direkt in die ausbildenden Universitäten hinein. Die Frage, inwiefern dies eine Gefährdung der grundgesetzlich verbürgten Autonomie der Hochschulen darstellt, wird durchaus kontrovers diskutiert (zur Autonomie der Hochschulen vgl. Abschnitt 3.1, Punkt 5). Als obligatorisch zu lehrende thematische Felder benennt die KMK (2004) u. a.: Bildung und Erziehung, Beruf und Rolle des Lehrers, Umgang mit berufsbezogenen Konflikt- und Entscheidungssituationen, Didaktik und Methodik, Lernen, Entwicklung und Sozialisation bis hin zu Schulentwicklung und Bildungsforschung.

Die durch die KMK verabschiedeten Konzepte werden in den Bundesländern zunehmend im Sinne ordnungspolitischer Rahmen weiterentwickelt. Vorangetrieben wird dies vor allem durch die Schuladministration der Bundesländer (für das Bundesland Berlin vgl. das Grundlagenpapier 2006). Bisher nur wenig beachtet wird die Tendenz, die Kompetenzorientierung faktisch - nicht jedoch von ihrer Benennung her in Richtung auf performanzorientierte Konzepte zu verändern; letztere beinhalten einen hohen normativen 
Anspruch vor allem für die Ausbildungsphase des Vorbereitungsdienstes. Dies umfasst zweierlei: Auf der einen Seite wird diese Performanzorientierung quasi ,verschleiert'; denn der verstärkte Rekurs auf das praktische pädagogische Handlungswissen im Sinne pädagogischer ,Meisterlehre' soll durch die modernisierte Sprachverwendung von Begriffen wie Kompetenz, Standards etc. anschlussfähig an wissenschaftlich basiertes Wissen gemacht werden (kritisch vgl. z. B. van Buer, Kohring \& Frasch 2009: 61ff.); dadurch soll dem erfahrungsbasierten Handlungswissen auch eine erhöhte Wertigkeit verliehen werden. Zum anderen sollen diese Begriffe als wissenschaftlich legitimierte Konzepte den Rahmen für handlungspraktische Normierungen bezüglich normativer Vorstellungen darüber abgeben, was unter ,gutem Unterricht zu verstehen sei und was nicht. Die Verstärkung der praxisorientierten Anteile bereits in der ersten Phase der Berufsausbildung fokussiert den Blick der Studierenden derzeit erkennbar auf die Frage, inwiefern das zu erlernende Wissen als handlungspraktisch erkannt wird und verwertbar sei (vgl. Abschnitt 3.3.3). Diese Verstärkung von practical knowledge - knowledge in action (vgl. Abschnitt 2.1.5) verstärkt die Gefahr für die Studierenden, sich in ihrer professionellen Biographie mit der subjektiven Präferenz von ,pädagogischer Meisterlehre' zumindest unbewusst bereits im Studium in die Richtung zu entwickeln, die Helsper (1996: 535) als „routinisierte Erstarrung“ bezeichnet (genauer vgl. Abschnitt 2.1.2).

\subsubsection{Praxisphasen in der deutschen Lehrerbildung - empirische Befunde}

Zur allgemeinen Einschätzung der empirischen Befundlage: Zunächst ist festzuhalten: Die empirische Befundlage zur Wirksamkeit der Praxisphasen in der universitären Lehrerbildung bezüglich des Aufbau professionellen Handlungswissens kann nur als spärlich gekennzeichnet werden (vgl. z. B. Hascher 2012). Nochmals verstärkt gilt dies hinsichtlich der Wirksamkeit bezüglich professioneller Handlungssteuerung im Unterricht selbst. Nicht überraschend ist weiterhin: Bezüglich der Ausweitung der Praxisphasen in der universitären Lehrerbildung durch die Einführung des so genannten Praxissemesters liegen ebenfalls nur wenige empirisch gesicherte Ergebnisse vor. Zudem weisen die wenigen verfügbaren empirischen Studien eine ausgesprochen heterogene, in Teilen auch widersprüchliche Befundlage aus. Die große Mehrheit der empirischen Studien zur Wirksamkeit der ,Praxis'phasen zielt auf die Beantwortung der folgenden Frage: Inwiefern haben diese Phasen die Zufriedenheit der Studierenden mit ihrem Studium, ihrer Berufswahl etc. verstärkt? Erst jüngst werden Untersuchungen zum Aufbau professionellen Wissens vorgelegt. Studien zur reflektierten Verknüpfung von theorieorientiertem Wissen einerseits und erfahrungsgestütztem Wissen und hinsichtlich der Wirksamkeit dieser beiden Wissenssorten bezüglich der Steuerung professionellen Handelns im Unterricht als Ort konfligierender Interessenstrukturen bzw. als ill defined problem liegen bisher nicht vor (vgl. Abschnitte 2.1.2 und 2.1.3), zumindest nicht für die deutsche Lehrerbildung. Somit bleibt die Frage weitestgehend unbeantwortet, inwiefern die Ausweitung der ,Praxis'phasen im universitären Studium zu einem Anstieg reflektierter Aneignung professionellen Handelns beiträgt.

Gleichwohl ist ebenfalls festzustellen: Trotz der durchaus begrenzten Aussagekraft der vorliegenden Befundlage und trotz der teilweise widersprüchlichen empirischen Evidenzen (vgl. z. B. Brouwer 2010; Offenberg \& Walke 2013; Tittle 2006) herrscht in der einschlägigen Diskussion generell die Einstellung vor - umgangssprachlich formuliert: „Viel hilft viel“: So seien Praktika bereits aus sich selbst heraus wirkungsvoll; sie trügen zweifelsfrei zur Professionalisierung angehender Lehrkräfte bei. Wenn kontroverse Debatten über eine Ausweitung der Praxisphasen in der universitären Lehrerbildung stattfinden, werden diese beinah ausschließlich auf der organisatorisch-verfahrenstechnischen Ebene geführt, also im Bereich der Definition von Kreditpunkten und Modulstrukturen im Gesamtrahmen der Studiengänge (vgl. z. B. die kritische Diskussion in Offenberg \& Walke 2013: 106). Sicherlich ein wenig überspitzt formuliert: Das grundsätzliche In-Frage-Stellen der Praxisphase scheint in der deutschen Diskussion beinah tabuisiert. Vor diesem Hintergrund stellt sich damit die kritische Frage nach dem Ideologiegehalt dieser Debatte.

Bereits in den traditionellen Lehramtsstudiengängen, die mit dem 1. Staatsexamen endeten und die 2004-2006 durch das neue Studiengangskonzept ersetzt wurden, führte das ,Theorie-Praxis'-Verhältnis (vgl. Abschnitt 2.1.4), das nur in Ansätzen diskutiert und in den universitären Curricula nur ansatzweise systematisch behandelt wurde, zu signifikanten Übergangsproblemen der Studierenden in den Vorbereitungsdienst. Diesen Übergang beschrieben die ehemaligen Studierenden wesentlich als "Praxisschock": Die staatlichen Ausbildungsakteure in dieser Phase agierten - so ein geflügeltes Wort im Vorbereitungsdienst - mit der folgenden Empfehlung: „Vergesst jetzt alles, was Ihr in der Universität gelernt habt. Hier im Referendariat erlebt Ihr die Wirklichkeit und was wirklich wichtig ist". Allerdings scheint es, als 
bliebe auch im neuen Bachelor-Master-Modell der Lehrerbildung das Problemfeld der reflektierten Verknüpfung von ,Theorie' und ,Praxis' sowohl in den Praxisphasen des Studiums selbst als auch beim Übergang in den Vorbereitungsdienst erhalten (vgl. Vogel 2011: 3). Denn jenseits der Neuregelung von zeitlichen und organisatorischen Rahmungen bleibt oft unklar, welches institutionelle Unterstützungshandeln es den Studierenden ermöglichen soll, sich konstruktiv-kritisch, gegebenenfalls auch konfrontativ mit der versprochenen bzw. faktischen Nutzbarkeit der von innen erworbenen Wissensrepertoires in der ,Praxis', d. h. in Schule und Unterricht, auseinanderzusetzen. Offenberg \& Walke (2013: 22) formulieren diesen Zustand wie folgt: "Die Leitlinien und übergeordneten Zielsetzungen werden formuliert, der Weg zum Ziel jedoch nicht ansatzweise deutlich".

Zu empirischen Einzelbefunden - Befunde zur Wirksamkeit der Praxisphasen auf die Studienzufriedenheit: Die meisten Studien fokussieren die Wirksamkeit der ,Praxis'phasen, indem sie die Zufriedenheit der Studierenden als Output-Parameter messen. Die aktuelle und durchaus heterogen strukturierte Befundlage kann wie folgt zusammengefasst werden: In ihrem Rückblick weist die große Mehrzahl der Studierenden diesen Phasen, vor allem dem Praxissemester, eine große bis sehr große Bedeutung für das Lehramtsstudium zu. Gleichzeitig begrüßen sie es nach wie vor, dass das Studium auch weiterhin eher auf eine theoretische Fundierung hin ausgerichtet ist (vgl. Holtz 2014: 100); allerdings weisen hier die Meinungen eine vergleichsweise hohe Varianz aus. Ihre Kompetenzen, Unterricht bewältigen zu können, schätzen die Studierenden als hoch bis sehr hoch ein; dabei beziehen sie sich vor allem auf ihr fachwissenschaftliches und auf ihr fachdidaktisches Wissen (vgl. Böttcher \& Blasberg 2015: 15; Holtz 2014: 101; Hascher, Baillod \& Wehr 2004). Allerdings beschreiben sie die Verknüpfung beider Wissensrepertoires als eher schwierig. Gleichzeitig empfinden die Studierenden die in den Fachwissenschaften erworbenen Wissensrepertoires und das für das praktische Handeln in Unterricht nutzbare Wissen als stark voneinander getrennte Welten (vgl. Kuhlee, van Buer \& Klinke 2009: 191; Böttcher \& Blasberg 2015: 15). Ähnliches gilt auch für die Vor- und Nachbereitungsveranstaltungen der ,Praxis'phasen, die curricular in den fachdidaktischen Studienanteilen platziert sind. Insgesamt fühlt sich die Mehrheit der Studierenden durch die innen angebotenen Studieninhalte nur unzureichend auf die Anforderungen vorbereitet, auf die sie im Unterricht und dann in ihrer späteren Berufspraxis treffen (vgl. Böttcher \& Blasberg 2015: 15; Holtz 2014: 109f.). Holtz (2014) zeigt auch, welche Art von Wissen die Mehrheit der Studierenden als "praxisrelevant“ verstehen: Es geht um „Tipps und Tricks", die versprechen, zur Bewältigung des unterrichtlichen Alltags beizutragen. Studieninhalte, die auf die reflektierte Aneignung späteren professionellen Handelns zielen, deklarieren viele Studierenden als "praxisfern“ oder sogar als "sinnlos“. Folgt man Kuhlee, van Buer \& Klinke (2009: 176ff.), richten sich die Erwartungen durchaus einer Mehrheit der Studierenden des Lehramts darauf, in den berufswissenschaftlichen Studieninhalten sollten die Grundelemente einer differenzierten Praxeologie als Basis für späteres berufliches Handeln gelehrt werden. So ist es nicht überraschend, dass Kuhlee, van Buer \& Klinke (2009: 190f.) für das erste Praktikum, das bereits im Bachelorstudium stattfindet, feststellen: Die Studierenden geraten in eine „Authentizitätsfalle“ bezüglich des im Studium als erfolgsrelevant deklarierten, wissenschaftlich fundierten Wissens auf der einen Seite und des erfahrungsgebundenen Wissens auf der anderen Seite, das sie als direkt relevant für die Problembewältigung im Unterricht erleben. In der Folge wünschen sich die Studierenden für ihr weiteres Studium eine intensivere Betreuung und Unterstützung seitens der Universität während der ,Praxis'phasen sowie eine stärkere Berücksichtigung pädagogisch-didaktischer Themen, dabei vor allem deren ,praxisnahe' Aufbereitung (vgl. Böttcher \& Blasberg 2015; Holtz 2014).

Zu Befunden der Wirksamkeit der ,Praxis'phasen auf den Aufbau unterrichtsrelevanten Wissens - die Selbstsicht der Studierenden: Die vorliegenden Befunde greifen in der Regel auf Daten zur Selbsteinschätzung der betroffenen Studierenden zurück, in Teilen auch auf solche der Lehrenden (z. B. Jantowski 2010; Schmitt \& Gröschner 2010). Schmitt (2009) kann für seine Studie zeigen: Bezüglich der Ausweitung der Praxisanteile während der universitären Phase stellt sich eine signifikante Zunahme der von den Studierenden wahrgenommenen Kompetenzen in „Unterrichten“, „Erziehen“, „Beurteilen“ und „Innovieren" ein. Allerdings sind diese Effekte von maximal mittlerer Effektstärke und in Teilen instabil. Ähnliches berichten auch kleinere Studien (z. B. Nölle 2002; Fölling-Albers u. a. 2005). Weitere Studien wie diejenigen von Nölle (2002) und Oelkers (2007) weisen positive Auswirkungen einer ausgeprägten Behandlung von ,Theorie-Praxis'-Erfahrungen während des universitären Ausbildung auf das unterrichtsrelevante pädagogische Wissen der Studierenden nach, zumindest aus der Sicht der Studierenden; allerdings verbleiben auch hier die Effektstärken im Bereich geringer und maximal mittlerer Stärke. Hascher 
(2012: 89) macht auf Ergebnisse von Wissensaufbau aufmerksam, das im Sinne aufgeklärter Aneignung von ,Praxis' als eher prekär einzuschätzen ist: Danach neigen Studierende in ihren Praktika dazu, eher unreflektiert Handlungsmuster zu übernehmen, die sie bei den von ihnen beobachteten Lehrkräften als erfolgreich wahrgenommen haben. Damit begrenzen sie ihre Möglichkeiten, über reflektierte professionelle Entwicklungsprozesse Handlungsmuster zur Generierung bestmöglicher ,Praxis' aufzubauen (vgl. auch Chitpin, Simon \& Galipeau 2008; Wideen, Mayer-Smith \& Moon 1998). So ist es nicht überraschend, dass in Praktika in bedeutsamem Ausmaß Anpassungsleistungen zu beobachten sind, die stark subjektiv und vor allem kontextspezifisch geprägt sowie auch weniger reflektiert sind (z. B. Schumacher \& Lind 2000; bereits Terhart 1990; Ulich 1996). Dennoch werden Praktika von den Studierenden weitgehend unabhängig von der dort jeweils erlebten Qualität als positiv beurteilt; denn aus ihrer Sicht eröffnen sie innen die Möglichkeit, unterrichtliches Handeln selbst ,auszuprobieren' (vgl. z. B. Schumacher \& Lind 2000). Ferner sieht sich die Mehrzahl der Studierenden in ihren Vorstellungen bestätigt, mit dem Beruf des Lehrers bzw. der Lehrerin das ,richtige' spätere Tätigkeitsfeld gewählt zu haben; ein Effekt ist, dass sie sich in ihrem Berufswunsch bestärkt sehen (vgl. Kuhlee, van Buer \& Klinke 2009: 183). In seinen Überlegungen zum Theorie-Praxis-Bezug in der Lehrerbildung macht Hedtke (2000: 6f.) auf Folgendes aufmerksam: Schulpraktische Übungen stellen für die Studierenden nicht die „Praxis des beruflichen Alltags, sondern die Praxis des seltenen Besuchs" dar. Müller (2010: 68f.) nimmt dieses Argument auf; sie verweist darauf, dass die Schulpraktika nicht die „affektgeprägte Betroffenenperspektive (..) erzeugen, sondern eine distanzierte Beobachtungsperspektive (..) ermöglichen“. Weiterhin ist festzuhalten: Während hinsichtlich der Effekte der ,Praxis'phasen auf die Entwicklung von Studierenden zumindest partiell empirische Studien vorliegen, ist dies bezüglich der Wirkungen auf Ausbildungsinstitutionen so gut wie nicht der Fall. Hascher (2012: 90) weist darauf hin, bisher lägen keine systematischen Evaluationen zu Praktika vor. Die Autorin interpretiert diesen Zustand wie folgt: Dieser Zustand sei Ausdruck eines relativ ,blinden' Glaubens an die Wirksamkeit der Praxis (vgl. bereits Brunner 1985); er sei der eingangs dieses Abschnittes bereits angesprochene Glauben in den "Mythos Praktikum" (vgl. Hascher 2011). Gestützt wird er durch den bildungs- und hochschulpolitischen Druck, handlungspraktische Phasen in Schule und Unterricht in größerem Umfang als Teil des universitären Studiums zu etablieren (vgl. auch Abschnitt 3.3.1).

Studien zum Aufbau professionellen Wissens: Insgesamt ist national wie auch international der Trend zu beobachten, unter Bezug auf Kompetenzmodelle und darauf basierende Messmodelle die Wirksamkeit der Berufsausbildung von Lehrkräften hinsichtlich des Aufbaus berufsrelevanten Wissens zu überprüfen. Darin eingeschlossen sind auch die ,Praxis'phasen (vgl. z. B. Blömeke, Kaiser \& Lehmann 2010; Klieme \& Leutner 2006). Diese Studien stellen Erkenntnisse zum Zusammenspiel verschiedener Wissensrepertoires der Befragten wie pädagogisches Wissen, fachdidaktisches Wissen und fachliches Wissen bereit; teils wird auch der Beitrag dieser Repertoires zum Prozess der Professionalisierung thematisiert. Forschungsprojekte wie TEDS-M (vgl. Blömeke, Kaiser \& Lehmann 2010), TEDS-LT (Blömeke, Bremerich-Vos, Haudeck, Kaiser, Nold, Schwippert \& Willenberg 2011) oder auch die Studie von Riese (2009) für das Fach Physik beschränken sich in ihren Erhebungen jedoch auf paper-and pencil-Tests, somit auf die Messung von Wissen; dessen faktische Handlungssteuerung für die Problemlösung für unterrichtliche Phänomene und Prozesse bleibt weitestgehend ausgeblendet. Einen ähnlichen Ansatz verfolgt die deutsche COACTIVStudie von Kunter, Baumert, Blum, Klusmann, Krauss \& Neubrand (2011) in Bezug auf die Lehrprofessionalität: Im Rahmen ihres zugrunde gelegten Kompetenzmodells weist sie korrelative Zusammenhänge zwischen mathematischem und mathematik-didaktischem Wissen und Lernerfolgen der von der Lehrkraft unterrichteten Schüler nach; diese sind allerdings von geringer Effektstärke. Die Frage, inwiefern das Wissen, über das die Lehrkraft verfügt, bzw. welche dieser Wissensrepertoires das unterrichtliche Handeln steuern, bleibt weitestgehend ungeklärt.

Empirische Befunde zum Praxissemester: Mittels einer Längsschnitt-Interventionsstudie geht Müller (2010: 100ff.) der Frage nach möglichen Effekten eines Praxissemesters nach (Praxisjahr an der Pädagogischen Hochschule Weingarten, Baden-Württemberg). Die Ergebnisse machen differentielle Effekte sichtbar: Sowohl bei der Interventions- als auch bei der (unbehandelten) Kontrollgruppe ist eine Zunahme an subjektiv wahrgenommenen Lehrkompetenzen festzustellen; allerdings sind im Vergleich der beiden Gruppen keine stabilen Effektrichtungen erkennbar. Dies betrifft auch den Aspekt der Selbstwirksamkeitserwartungen der Befragten. Müller fasst ihre Befunde wie folgt zusammen: Der ,Praxisbezug', der über Unterrichtspraktika bzw. über das Praxissemester vermittelt werde, scheine zwar generell zu einem höheren Selbstbewusstsein der Studierenden zu führen - hinsichtlich ihres Umgangs mit 
Schülern und Schülerinnen und hinsichtlich effektiver Unterrichtsplanung; allerdings sei dieser Effekt nicht unter allen Rahmenbedingungen eindeutig, und vor allem sei er zeitlich instabil.

\section{Praxisphasen in der deutschen Lehrerbildung - wenig Klärung des ,Theorie-Praxis-Problems' und ungeminderter Glaube an das Mythos Praktikum}

Die folgenden Ziellinien können für die deutsche Lehrerbildung als neue bzw. verstärkte Relevanzen festgehalten werden:

(a) Die Reform der Lehrerbildung stellt einen systemischen Bestandteil der Reform des Schulwesens dar:

Neu für Deutschland ist: Die Reform der Lehrerbildung in Deutschland ist als systemischer Bestandteil der Reform des deutschen Schulwesens, dort vor allem der Qualität der Einzelschule als pädagogischer Handlungseinheit, zu verstehen (vgl. Abschnitt 2.3). Ziellinie ist, die Wissensrepertories, die für die Umstellung von Schule und Unterricht auf kompetenzfokussierte Outputorientierung benötigt werden, den späteren Lehrkräften bzw. den Anwärtern zuverlässig bereits während ihrer Berufsausbildung, aber auch über die berufliche Weiterbildung zu vermitteln.

(b) In der universitären Lehrerbildung bleibt die Theorieorientierung zwar erhalten, wird aber durch eine signifikant erhöhte Praxisorientierung ergänzt:

Vor diesem allgemeinen Hintergrund wird derzeit in Deutschland die erneute Reform der Lehrerbildung implementiert. Während sich die erste Reform von 2004-2006 auf die Umstellung des einphasigen universitären Modells (1. Staatsexamen) auf das Bachelor-Master-Modell konzentrierte, thematisiert die jetzige Reform die Ausweitung und Neugestaltung der ,Praxis'anteile im universitären Studium. Im Unterschied zu Konzepten einer primär field-based teacher education, wie sie in den USA oder auch in UK zu finden sind (vgl. Abschnitt 2.1.5), soll die starke Theorieorientierung in der universitären Phase der Berufsausbildung der späteren Lehrkräfte erhalten bleiben. Diese bezieht sich nicht nur auf die fachwissenschaftlichen, sondern auch auf die berufswissenschaftlichen Studienanteile. Dabei ist für die Berufswissenschaften eine signifikante Ausweitung der ,Praxis'anteile festzustellen; dies ist der Zeitanteil, den die Studierenden während ihres Studiums in Schule und Unterricht verbringen. Gleichzeitig führt die Blockung dieser Anteile im Praxissemester dazu, dass die Praxis des "seltenen Besuchs" (vgl. Hedtke (2000: 6f.) durch die Praxis einer ca. dreimonatigen intensiven Erfahrung in Schule und Unterricht ersetzt wird. Dies ist eine Phase, in der die Studierenden (Beispiel Berlin) in jedem ihrer beiden Unterrichtsfächer jeweils 18 Unterrichtsstunden unterrichten sollen.

(c) Der „Mythos Praktikum“ wirkt auch weiterhin ungebrochen:

Die Frage, inwiefern über diese Veränderungen das im Abschnitt 2.1.4 als durchaus prekär beschriebene Verhältnis von ,Theorie' und ,Praxis' durch die erhöhte Praxisorientierung aufgeklärt und die bisher beobachtete Problemlage des „Praxisschocks" zumindest gemindert werden, kann derzeit empirisch basiert nicht eindeutig beantwortet werden. Auffällig ist: Vor allem seitens der Akteure aus der Bildungspolitik, aber auch seitens einer großen Anzahl von Akteuren in den Hochschulen, ist der Glaube an das "Mythos Praktikum" (Hascher 2011) weiterhin ungebrochen.

(d) Die implementierte Praxisorientierung wirkt nicht, automatisch' in Richtung einer aufgeklärten Aneignung von Praxis:

Der derzeitige Stand der einschlägigen Befunde zu den Praxisphasen in der deutschen universitären Lehrerbildung kann wie folgt zusammengefasst werden: Für die Studierenden ist der Stellenwert der Praktika hoch; allerdings nimmt er im Verlauf des Studiums ab. Folgt man Kuhlee, van Buer \& Klinke (2009: 170ff.), koppeln ,Praxis'erfahrungen an die generellen ,praxisorientierten' Erwartungshaltungen der Studierenden im Lehramt an. Neben einer guten fachwissenschaftlichen Ausbildung wollen sie vor allem eine ,gute' Ausbildung als spätere Lehrkraft erhalten - und diese solle bereits in der universitären Ausbildung umfangreiche ,praktisch verwendbare' Wissensbestände enthalten (vgl. auch Müller 2010: 83ff.). Kuhlee, van Buer \& Klinke (2009: 125ff.) zeigen: Bereits bei der Berufswahl sowie über das gesamte Bachelorstudium hinweg empfindet sich beinah jeder zweite Studierende im Lehramt als "geborener Lehrer". Bezüglich der im Studium angebotenen erziehungswissenschaftlichen und (fach-) didaktischen 
Die ,neue' Lehrerbildung in Deutschland. Vom Praxisschock zur aufgeklärten Aneignung professionellen Handelns?...

Wissensrepertoires charakterisieren die Befragten sich eher als "theoriedistant". Vor diesem Hintergrund ist es nur folgerichtig, wenn Reinhoffer \& Dörr (2008) bzw. Weyland \& Wittmann (2011: 57) die einschlägigen Befunde zu den Praxisphasen in der universitären Lehrerbildung wie folgt auf den Punkt bringen: Die Praxisphasen, auch die Praxissemester, seien nicht ,automatisch' wirksam, zumindest nicht in Richtung der intendierten aufgeklärten Aneignung von Praxis. Erst in gesicherten Kontexten professioneller Vor- und Nachbereitung dieser Phasen sowie im Kontext intensiver Begleitung der Praktika selbst könnten nachhaltigere Effekte in der intendierten Richtung erwartet werden.

(e) Drängende Probleme wie die Abstimmung der fachwissenschaftlichen Inhalte auf die spätere Berufspraxis von Lehrkräften bleiben auch weiterhin ungelöst:

Die Forderung nach aufwändiger Vor- und Nachbereitung sowie Begleitung der ,Praxis'erfahrungen macht auf ein generelles Konstruktionsproblem deutscher Lehrerbildung aufmerksam; dieses gilt nicht nur für die Berufswissenschaften, sondern besonders auch für die fachwissenschaftlichen Inhalte. Studien zeigen, dass vor allem die akademische Integration von Lehramtsstudierenden über die fachwissenschaftlichen Inhalte des von ihnen gewählten Kernfaches verläuft, verbunden mit einem hohem Interesse am Lehrberuf, hoher Leistungsmotivation, stabiler Erfolgszuversicht und intrinsischer studienfachbezogener Motivation (vgl. van Buer \& Gausch 2011: 163). Gleichzeitig ist die Frage drängend, inwieweit die Curricula der akademischen Fächer die Aufgaben des zukünftigen Arbeitsplatzes Unterricht reflektieren. Denn die fachwissenschaftlichen Curricula sind nicht primär auf das Lehramt ausgerichtet, also z. B. auf das Unterrichtsfach Englisch. Statt dessen zielen sie auf die Vermittlung der fachwissenschaftlichen Inhalte z. B. in Anglistik; damit verknüpft ist der Anspruch, auf die Ausübung einer wissenschaftlich geprägten Tätigkeit in dem studierten Bereich vorzubereiten, gegebenenfalls in diesem Fach auch eine wissenschaftliche Karriere anzustreben. Nicht überraschend ist es zudem angesichts der reduzierten Zeitanteile, die die Studierenden des Lehramts in den jeweils zwei Fachwissenschaften im Vergleich zu den Studierenden von Monobachelor und Monomaster nutzen können: Lehramtsstudierende fühlen sich in den fachwissenschaftlichen Fächern häufiger nicht respektiert bzw. ernst genommen, sehen sich als „Studierende zweiter Klasse" tituliert (vgl. Böttcher \& Blasberg 2015: 19; Holtz 2014: 10). Gerade angesichts der Spannungen zwischen fachwissenschaftlicher Ausrichtung des universitären Studiums und Erwartungen der Studierenden an das Studienangebot verweisen Offenberg \& Walke $(2013,110)$ auf die folgende Gefahr: Die Inhalte und Methoden der universitären Phase sind aufgrund eines ökonomisch dominierten Verständnisses von Outputeffektivität und -effizienz zu schnell und eindeutig an den Ausbildungsbedürfnissen des staatlichen Vorbereitungsdienstes ausgerichtet. Rischke, Bönsch \& Müller (2014) hingegen plädieren für die massive Verstärkung der ersten und zweiten Phase; allerdings verweisen sie auch darauf, die Erwartungen, Rollen und Zuständigkeiten neu zu klären und über die Curricula sichtbar zu machen. Denn der äußerst straffe Studienfahrplan - so Rischke, Bönsch \& Müller (2014: 11f.) - lasse kaum Raum für die institutionell unterstützte aufgeklärte Aneignung von ,Praxis'.

(f) Gezielte Kompetenzorientierung der universitären Lehrerbildung im Sinne der Vermittlung Evidenz basierter Praxis als Problemlösung?

Die Ausweitung der berufswissenschaftlichen Studienanteile führt zu der drängenden Frage, inwiefern der beschriebene Zugewinn an ,Praxis' curricular und hochschuldidaktisch genutzt werden kann, um den Studierenden besser als bisher einen gesicherten Zugang zu den Wissensrepertories zu eröffnen, die als zentral für professionelles Lehrhandeln angesehen werden. Geisteswissenschaftlich gesprochen, geht es um die aufgeklärte Aneignung von professionellem Denken und Handeln. Die Beiträge in Huber, Hellmer \& Schneider (2009) weisen aus, wie der Zugang zu dieser Art pädagogischer und fachdidaktisch basierter Theorie durch forschendes Lehren verbessert werden kann. Ähnliches zeigen die Beiträge in Roters, Schneider, Koch-Priewe, Thiele \& Wildt (2009): Hier stehen schul- und unterrichtsbezogene Projekte im Zentrum; mit ihrer Hilfe soll die „systemische Zwitterstellung universitär begleiteter und verantworteter schulischer Praxisphasen" (Schneider \& Wildt 2009: 8) erkennbar verringert werden. Gleichzeitig geht es jedoch auch darum, die typologische Differenz der Wissensformen zwischen wissenschaftlich fundiertem Wissen und praktischem Handlungswissen nicht einzuebnen. Ein entscheidender Unterschied zu den performanzorientierten Konzepten, wie sie bereits im Microteaching (z. B. Ward 1970) etc. in den 1960er und 1970er Jahren favorisiert wurden und wie sie ebenfalls in Konzepten der field-based teacher education (vgl. Abschnitt 2.1.4) zu finden sind, liegt in der gezielten Kompetenzorientierung der Ansätze zum forschenden Lernen. Für diese Form hochschuldidaktischer Konzeptionierung stellt sich nach wie vor die nur 
unzureichend geklärte Frage nach der systematischen Verwendung des erworbenen wissenschaftlichen Wissens für professionelles Handeln in Schule und Unterricht. Neuere Studien (vgl. z. B. Klein, Wagner, Klopp \& Stark: 2015) bzw. laufende Projekte (vgl. z. B. Zeuch \& Souvignier: 2015) versuchen, im Rahmen des Konzepts Evidenz basierter Lehrerbildung die mehrfach angesprochene Spannung zwischen ,Theorie und Praxis' in der deutschen Lehrerbildung wenn schon nicht zu lösen, so doch zu mindern.

Folgt man dem Konzept Evidenz basierter Lehrerbildung, geht es darum, das universitäre Curriculum wesentlich wie folgt aufzubauen: Die Studierenden sollen lernen, die bildungswissenschaftlich vorliegenden, vor allem die verfügbaren empirischen Befunde zu Schule und besonders zu Unterricht konsistent argumentierend in ihre Handlungsplanung zu integrieren. In den universitären Seminaren ist dies zunächst eine entworfene Planung; während ihrer Praktika wird diese Planung zu einer ,Theorie-Praxis' gekoppelten Handlungsplanung und -umsetzung. Im Sinne von Scientific Reasoning (vgl. Fischer, Kollar et al.: 2014) geht es darum, in den Studienprogrammen zur Lehrerbildung systematisch die Kompetenz der Studierenden aufzubauen, mit wissenschaftlicher, vor allem empirisch fundierter Evidenz bezüglich ihrer (unterrichtlichen) Handlungsentwürfe zu argumentieren und dies über reflektierte Heuristiken zu vervollständigen (vgl. Wenglein, Bauer, Heininger \& Prenzel 2015: 211ff.). Damit könnte ein wichtiger Schritt in Richtung konsistentes, wissenschaftlich basiertes Argumentieren der späteren Lehrkräfte erreicht werden. Die Frage allerdings, inwiefern dieses eine signifikante Ausgangsbasis für das operative Handeln im Unterricht selbst darstellt und die nachhaltige Verbesserung der ex-post-facto-Reflektion über den vollzogenen Unterricht sichert, bleibt auch weiterhin offen.

\section{References}

Ackeren, van, I. \& Brauckmann, S. (2010). Internationale Diskussions-, Forschungs- und Theorieansätze zur Governance im Schulwesen. In: Altrichter, H. \& Maag Merki, K. (Hrsg.), Handbuch Neue Steuerung im Schulsystem (pp. 41-61). Wiesbaden: VS Verlag für Sozialwissenschaften.

Ahrbeck, B. (2014). Inklusion. Eine Kritik. Stuttgart: Kohlhammer.

Altrichter, H. \& Brüsemeister, T. (2012). Educational Governance: Handlungskoordination und Steuerung im Bildungssystem. Wiesbaden: VS Verlag für Sozialwissenschaften.

Altrichter, H. \& Helm, C. (2011). Schulentwicklung und Systemreform. In: Altrichter, H. \& Helm, C. (Hrsg.), Akteure und Instrumente der Schulentwicklung (pp. 13-35). Baltmannsweiler: Schneider Verlag Hohengehren.

Altrichter, H., Heinrich, M. \& Soukup-Altrichter, K. (2011). Schulentwicklung durch Schulprofilierung? Zur Veränderung von Koordinationsmechanismen im Schulsystem. Wiesbaden: VS Verlag für Sozialwissenschaften.

Arnold, R. \& Prescher, T. (Hrsg.) (2014). Schulentwicklung systemisch gestalten: Wege zu einem lebendigen und nachhaltigen Lernen in Schule und Unterricht. Köln: Link.

Auernheimer, G. (Hrsg.) (2006). Schieflagen im Bildungssystem. Die Benachteiligung der Migrantenkinder. Wiesbaden: VS Verlag für Sozialwissenschaften.

Barz, H. (2010). Bildungsmarketing. In: Barz, H. (Hrsg.), Handbuch Bildungsfinanzierung (pp. 415-427). Wiesbaden: Springer Gabler.

Bauer, J., Prenzel, M. \& Renkl, A. (2015). Evidenzbasierte Praxis - im Lehrerberuf?! Einführung in den Thementeil. Unterrichtswissenschaft, 43. Jahrgang, Heft 3, pp. 188-192.

Baum, E. (2014). Kooperation und Schulentwicklung. Wie Lehrkräfte in Gruppen Entwicklungsanlässe bearbeiten. Wiesbaden: Springer VS. 
Die ,neue' Lehrerbildung in Deutschland. Vom Praxisschock zur aufgeklärten Aneignung professionellen Handelns?...

Baumert, J. \& Kunter, M. (2011). Das Kompetenzmodell von COACTIV. In: Kunter, M., Baumert, J. u.a. (Hrsg.), Professionelle Kompetenz von Lehrkräften. Ergebnisse des Forschungsprogramms COACTIV (pp. 29-53). Münster et al.: Waxmann.

Berkemeyer, N. (2010). Die Steuerung des Schulsystems. Theoretische und praktische Explorationen. Wiesbaden: VS Verlag für Sozialwissenschaften.

Besser, M. \& Krauss, S. (2009). Zur Professionalität als Expertise. In: Zlatkin-Troitschanskaia, O., Beck, K. u. a. (Hrsg.), Lehrprofessionalität. Bedingungen, Genese, Wirkungen und ihre Messung (pp. 71-82). Beltz: Weinheim und Basel.

Beyer, K. (2014). Didaktische Prinzipien: Eckpfeiler guten Unterrichts: Ein theoriebasiertes und praxisorientiertes Handbuch in Tabellen für den Unterricht auf der Sekundarstufe II. Hohengehren: Schneider.

Blömeke, S. (2006). Struktur der Lehrerausbildung im internationalen Vergleich. Ergebnisse einer Untersuchung zu acht Ländern. Zeitschrift für Pädagogik, Jahrgang 52, Heft 3, 393-416.

Blömeke, S., Kaiser, G. \& Lehmann, R. (2010). Professionelle Kompetenz und Lerngelegenheiten angehender Mathematiklehrkräfte für die Sekundarstufe I im internationalen Ländervergleich. Münster: Waxmann.

Blömeke, S., Bremerich-Vos, A., Haudeck, H., Kaiser, G., Nold, G., Schwippert, K. \& Willenberg, H. (Hrsg.) (2011). Kompetenzen von Lehramtsstudierenden in gering strukturierten Domänen. Erste Ergebnisse aus TEDS-LT. Münster: Waxmann.

Böttcher, W. (2006). Bildungsstandards und Evaluation im Paradigma der Outputsteuerung. In: Böttcher, W., Holtappels, H. G. \& Brohm, M. (Hrsg.), Evaluation im Bildungssystem. Eine Einführung in Grundlagen und Praxisbeispiele (pp. 39-48). Weinheim \& München: Juventa.

Böttcher, W. \& Blasberg, S. (2015). Strategisch aufgestellt und professionell organisiert? Eine explorative Studie zu Strukturen und Status der Lehrerbildung. Bonn: Deutsche Telekom Stiftung.

Bohl, T., Helsper, W., Holtappels, H., G. \& Schelle, C. (Hrsg.) (2010). Handbuch Schulentwicklung. Bad Heilbrunn: Klinkhardt.

Bohl, T., Harant, M. \& Wacker, A. (2014). Schulpädagogik und Schultheorie. Stuttgart: UTB.

Bosse, D. (2012). Zur Situation der Lehrerbildung in Deutschland. Reform der Lehrerbildung, Heft 5, pp.1-16.

Brunner, R. (1985). Der Einsatz praxisorientierter Verfahren in der Lehrerbildung an den Hochschulen der Bundesrepublik Deutschland. Unterrichtswissenschaft, 2, pp. 169-181.

Brouwer, C. N. (2010). Determining Long Term Effects of Teacher Education. In: Penelope, P., Eva, B. \& Barry, M. (Ed.), International Encyclopedia of Education (pp. 503-510). Oxford: Elsevier.

Buer, J. van (1990). Pädagogische Freiheit des Lehrers im unterrichtlichen Alltag. Realität oder Illusion? Frankfurt a. M., Bern, New York \& Paris: Peter Lang Verlag.

Buer, J., van, Kohring, A. \& Frasch, F. (2009). Die ,neue' Lehrerbildung an der Humboldt-Universität 2004-2009 - eine kritische evaluationsgestützte Stellungnahme. Studien zur Wirtschafts- und Berufsbildungsforschung aus der Humboldt-Universität zu Berlin. Band 17. Berlin: Humboldt-Universität.

Buer, J., van \& Gausch, M. (2011). Wechselhandeln von Studierenden in den lehramtsbezogenen Bachelorstudiengängen der Humboldt-Universität. Studien zur Wirtschafts- und Berufsbildungsforschung aus der Humboldt-Universität zu Berlin. Band 22. Berlin: Humboldt-Universität. 
Buer, J. van, Wagner, C., Rückmann, J. \& Füssel, H.-P. (2015). Schulinspektion - zur prekären Balance zwischen Instrumentatiliät versus Professionalität in der Regelinspektion. In: Rausch, A., Warwas, J., Seifried, J. \& Wuttke, E. (Hrsg.), Konzepte und Ergebnisse ausgewählter Forschungsfelder der beruflichen Bildung - Festschrift für Detlef Sembill (pp. 337-357). Baltmannsweiler: Schneider Verlag Hohengehren $\mathrm{GmbH}$.

Bund-Länder-Vereinbarung (2013). Bund-Länder-Vereinbarung über ein gemeinsames Programm "Qualitätsoffensive Lehrerbildung" gemäß Artikel 91b des Grundgesetzes. 12. April 2013. Berlin.

Burstow, B. (2015). Studying and Learning in Initial Teacher Education in England. In: Kuhlee, D., Buer, J. van \& Winch, C. (Hrsg.), Governance in der Lehrerausbildung: Analysen aus England und Deutschland (Governance in Initial Teacher Education: Perspectives on England and Germany) (pp. 81-98). Wiesbaden: Springer VS.

Ceri, F. (2015). Die Bildungsbenachteiligung von Kindern mit Migrationshintergrund. Welche Folgen hat der schulische Umgang mit sprachlichen Differenzen auf die Bildungschancen? Wiesbaden: Springer VS.

Chin, C. A. \& Brewer, W. F. (1993). The role of anomalous data in knowledge acquisition: A theoretical framework and implications for science instruction. Review of Educational Research, 63 (1), pp. 1-49.

Chitpin, S., Simon, M. \& Galipeau, J. (2008). Pre-service teachers' use of the objective knowledge growth framework for reflection during practicum. Teaching and Teacher Education, 12, pp. 2049-2058.

De Boer, H., Enders, J. \& Schimank, U. (2007). On the way towards New Public Management? The Governance of University Systems in England, the Netherlands, Austria and Germany. In: Jansen, D. (Ed.), New Forms of Governance in Research Organisations (pp. 137-152). Dordrecht: Springer.

Deming, W. E. (1982). Productivity and Competence Position. Cambridge: UP.

Deutsche Telekom Stiftung (2015). Lehrerbildung - Verantwortung für die Zukunft. Herausforderungen und Perspektiven. 11. Juni 2015. Eine Veranstaltung von Deutsche Telekom Stiftung, Hochschulrektorenkonferenz und Stifterverband für die Deutsche Wissenschaft. Abrufbar unter: www.hrk.de/themene/lehre/arbeitsfelder/lehrerbildung-tagung-2015/

Döbert, H. \& Dedering, K. (2008). Externe Evaluation von Schulen in Deutschland - die Konzepte der Bundesländer, ihre Gemeinsamkeiten und Unterschiede. In: Döbert, H. \& Dedering, K. (Hrsg.), Externe Evaluation von Schulen. Historische, rechtliche und vergleichende Aspekte (63-151). Münster: Waxmann.

Dupriez, V. \& Maroy, C. (2003). Regulation in school systems: A theoretical analysis of the structural framework of the school system in French-speaking Belgium. Journal of Education, Heft 4, pp. 375-392.

Eisinger, B., Warndorf, P. K., Falterbaum, J. \& Feldt, J. (2010). Bildungsfinanzierung: Studie zu Finanzierungsmodellen für das deutsche Schulsystem. Münster: Waxmann.

Ehmke, T., Klieme, E. \& Stanat P. (2013). Veränderungen der Lesekompetenz von PISA 2000 nach PISA 2009. Zeitschrift für Pädagogik. Beiheft. Jahrgang 59, pp. 132-150.

Ertl, H. (2015). Partnership-based Initial Teacher Education in England: A Discussion of the Oxford Internship Scheme in the Light of Past and Current Reform Agendas. In: Kuhlee, D., Buer, J. van \& Winch, C. (Hrsg.), Governance in der Lehrerausbildung: Analysen aus England und Deutschland (Governance in Initial Teacher Education: Perspectives on England and Germany) (pp. 81-98). Wiesbaden: Springer VS.

Fauser, P. (1986). Pädagogische Freiheit in Schule und Recht. Weinheim \& Basel: Beltz. 
Die ,neue' Lehrerbildung in Deutschland. Vom Praxisschock zur aufgeklärten Aneignung professionellen Handelns?...

Fend, H. (2009). Neue Theorie der Schule. Einführung in das Verstehen von Bildungssystemen. Wiesbaden: VS Verlag für Sozialwissenschaften.

Fischer, F., Kollar, I., Ufer, S. et al. (2014). Scientific reasoning and argumentation: Advancing an interdisciplinary research agenda in education. Frontline Learning Research, vol. 3, 1-8.

Fölling-Albers, M. u. a. (2005). Diagnose- und Förderkompetenzen erwerben - „Situierte Lernbedingungen“. journal für lehrerlnnenbildung, Heft 5, pp. 54-63.

Füssel, H.-P. \& Buer, J. van (2015). Should Schoolteachers in Germany be Tenured Civil Servants, and is there Something „Typically German“ About this Issue? In: Kuhlee, D., Buer, J. van \& Winch, C. (Hrsg.), Governance in der Lehrerausbildung: Analysen aus England und Deutschland (Governance in Initial Teacher Education: Perspectives on England and Germany) (pp. 131-147). Wiesbaden: Springer VS.

Grundlagenpapier (2006). Grundlagen für die Entwicklung lehramtsbezogener Masterstudiengänge Strukturvorgaben und Qualifikationsrahmen. Berlin. Abrufbar unter http://www.fuberlin.de/bms/doku/lehrer.pdf.

Hascher, T., Baillod, J. \& Wehr, S. (2004). Feedback von Schülerinnen und Schülern als Quelle des Lernprozesses im Praktikum von Lehramtsstudierenden. Zeitschrift für Pädagogik, 50 (2), pp. 223243.

Hascher, T. (2011). Vom "Mythos Praktikum" ... und der Gefahr verpasster Lerngelegenheiten. Journal für Lehrerinnen- und Lehrerbildung, 3, pp. 8-16.

Hascher, T. (2012). Forschung zur Bedeutung von Schul- und Unterrichtspraktika in der Lehrerinnen- und Lehrerbildung. Beiträge zur Lehrerbildung, 30 (1), pp. 87-98.

Hattie, J. (2009). Visible learning. London \& New York: Routledge.

Hedtke, R. (2000). Das unstillbare Verlangen nach Praxisbezug - Zum Theorie-Praxisproblem der Lehrerbildung am Exempel Schulpraktischer Studien. Abrufbar unter http://www.sowionlinejournal.de/lehrerbildung/hedtke.htm.

Hedtke, R. (2007). Das Studium als vorübergehende Unterbrechung der Schulpraxis. Anmerkungen zur geschlossenen Welt der Lehrerausbildung. In: Kostrzewa, F. (Hrsg.), Lehrerbildung im Diskurs (pp. 25-89). Berlin: LIT-Verlag.

Heid, H. (2007). Qualität von Schule - Zwischen Bildungstheorie und Bildungsökonomie. In: Buer, J. van \& Wagner, C. (Hrsg.), Qualität von Schule. Ein kritisches Handbuch (pp. 55-66). Frankfurt a. M.: Lang.

Heinrich, M. (2008). Von der Steuerung zu Aushandlungsprozessen als neue Form der Handlungskoordination. In: Langer, R. (Hrsg.), ,Warum tun die das?' Governanceanalysen zum Steuerungshandeln in der Schulentwicklung (pp. 31-46). Wiesbaden: VS Verlag für Sozialwissenschaften.

Heinrich, M. (2010). Bildungsgerechtigkeit durch Evidence-based-policy? Governanceanalysen zu einem bildungspolitischen Programm. In: Böttcher, W., Dicke, J. N. \& Hogrebe, N. (Hrsg.), Evaluation, Bildung und Gesellschaft. Steuerungsinstrumente zwischen Anspruch und Wirklichkeit (pp. 47-68). Münster et al.: Waxmann.

Heinrich, M. (2012). Governance in der Schulentwicklung: Von der Autonomie zur evaluationsbasierten Steuerung (Educational Governance). Wiesebaden: VS Verlag für Sozialwissenschaften.

Helsper, W. (1996). Antinomien des Lehrerhandelns in modernisierten pädagogischen Kulturen. Paradoxe Verwendungsweisen von Autonomie und Selbstverantwortlichkeit. In: Combe, A. \& Helsper, W. 
(Hrsg.), Pädagogische Professionalität. Untersuchungen zum Typus pädagogischen Handelns (pp. 521-569). Frankfurt a. M.: Suhrkamp Taschenbuch Verlag.

Hilligus, A. H. (2015). Structural Features and Particularities of Initial Teacher Education in Germany: The Specific Role of Universities. In: Kuhlee, D., Buer, J. van \& Winch, C. (Hrsg.), Governance in der Lehrerausbildung: Analysen aus England und Deutschland (Governance in Initial Teacher Education: Perspectives on England and Germany) (pp. 113-130). Wiesbaden: Springer VS.

Hochschulrektorenkonferenz (2006). Empfehlung zur Zukunft der Lehrerbildung in den Hochschulen. Bonn: HRK. Abrufbar unter www.krk.de/positionen/gesamtliste-beschluesse/position/ convention/empfehlung-zur-zukunft-der-lehrerbildung-in-den-hochschulen.

Hochschulrektorenkonferenz (2011). Entschließung „Zur Hochschulautonomie“. Entschließung der 10. Mitgliederversammlung am 3.5.2011. Bonn \& Berlin: HRK.

Holtz, P. (2014). „Es heißt ja auch Praxissemester und nicht Theoriesemester". Quantitative und qualitative Befunde. In: Kleinespel, K. (Hrsg.), Ein Praxissemester in der Lehrerbildung. Konzepte, Befunde und Entwicklungsperspektiven am Beispiel des Jenaer Modells (pp. 97-118). Bad Heilbrunn: Klinkhardt.

Holtappels, H. G. (2010). Schule als lernende Organisation. In: Bohl, T., Helsper, W., Holtappels, H., G. \& Schelle, C. (Hrsg.), Handbuch Schulentwicklung (pp. 99-105). Bad Heilbrunn: Klinkhardt.

Huber, L., Hellmer, J. \& Schneider, F. (Hrsg.) (2009). Forschendes Lernen im Studium. Aktuelle Konzepte und Erfahrungen. Bielefeld: UniversitätsVerlag Webler.

Jantowski, A. (2010). Universitätsprojekt „BiS“. Belastungen im Studium im Jenaer Modell der Lehrerbildung. Power Point Präsentation. Bielefeld (9. Februar).

Kitchener, K. (1983). Cognition, metacognition, and epistemic cognition: a three-level model of cognitive processing. Human Development, 26 (4), pp. 222-232.

Klein, M., Wagner, K., Klopp, E. \& Stark, R. (2015). Förderung anwendbaren bildungswissenschaftlichen Wissens bei Lehramtsstudierenden anhand fehlerbasierten kollaborativen Wissens: Eine Studie zur Replikation und Stabilität bisheriger Befunde und zur Erweiterung der Lernumgebung. Unterrichtswissenschaft, 43. Jahrgang, Heft 3, 225-244.

Kleinespel, K. (Hrsg.) (2014). Ein Praxissemester in der Lehrerbildung. Konzepte, Befunde und Entwicklungsperspektiven am Beispiel des Jenaer Modells. Bad Heilbrunn: Klinkhardt.

Klemm, K. \& Preuss-Lausitz, U. (2011). Auf dem Weg zur schulischen Inklusion in Nordrhein-Westfalen. Empfehlungen zur Umsetzung der UN-Behindertenrechtskonvention im Bereich der allgemeinen Schulen. Gutachten erstellt im Auftrag des Ministeriums für Schule und Weiterbildung des Landes Nordrhein-Westfalen. Essen \& Berlin (kl.klemm@t-online.de).

Klieme, E. \& Leutner, D. (2006). Kompetenzmodelle zur Erfassung individueller Lernergebnisse und zur Bilanzierung von Bildungsprozessen. Zeitschrift für Pädagogik, 52, pp. 876-903.

Klippert, H. (2000). Pädagogische Schulentwicklung. Planungs- und Arbeitshilfen zur Förderung einer neuen Lernkultur. Weinheim/Basel: Beltz.

Köller, M. (2009). Konstruktion und Implementierung von Schulprogrammen - Ein triangulativer Forschungsansatz. Frankfurt a. Main: Lang.

Köller, M. \& Pingel, R. (2015). Kommunikation für schulische Führungskräfte - Anregungen für die Praxis. München: Oldenbourg. 
Die ,neue' Lehrerbildung in Deutschland. Vom Praxisschock zur aufgeklärten Aneignung professionellen Handelns?...

Köller, O. (2007). Standards und Qualitätssicherung zur Outputsteuerung im System und in der Einzelinstitution. In: Buer, J. van \& Wagner, C. (Hrsg.), Qualität von Schule. Ein kritisches Handbuch (pp. 93-103). Frankfurt a. Main: Lang.

Köller, O., Möller, J. \& Möller, J. (2013). Was wirkt wirklich? Einschätzungen von Determinanten schulischen Lernens. München: Oldenbourg.

Kraler, C. \& Schratz, M. (Hrsg.) (2008). Wissen erwerben, Kompetenz entwickeln. Modelle zur kompetenzorientierten Lehrerbildung. Münster et al.: Waxmann.

Krauss, S. (2011). Das Experten-Paradigma in der Forschung zum Lehrerberuf. In: Terhart, E., Bennewitz, H. \& Rothland, M. (Hrsg.), Handbuch der Forschung zum Lehrerberuf (pp. 171-191). Münster et al.: Waxmann.

Krüger, H.-H., Deinert, A. \& Zschach, M. (Hrsg.) (2012). Jugendliche und ihre Peers. Freundschaftsbeziehungen und Bildungsbiografien in einer Längsschnittuntersuchung. Opladen et al.: Budrich.

Kuhlee, D. \& Buer, J. van (2009). Professionalisierung in der neuen gestuften Lehrerbildung - Zwischen traditionellen Berufsbildern der Studierenden und professionsorientierter Kompetenzentwicklung. In: Zlatkin-Troitschanskaia, O., Beck, K. u. a. (Hrsg.), Lehrprofessionalität. Bedingungen, Genese, Wirkungen und ihre Messung (pp. 489-499). Weinheim/Basel: Beltz.

Kuhlee, D., Buer, J. van \& Klinke, S. (2009). Strukturelle Studierbarkeit und Wirksamkeit der Lehrerbildung. Arbeitsbericht 2 zur Evaluation der Studiengänge Bachelor mit Lehramtsoption und Master of Education an der Humboldt-Universität zu Berlin. Studien zur Wirtschaftspädagogik und Berufsbildungsforschung aus der Humboldt-Universität zu Berlin. Bd. 13. Berlin: HumboldtUniversität zu Berlin.

Kuhlee, D., Buer, J. van \& Winch, C. (Hrsg.) (2015) Governance in der Lehrerausbildung: Analysen aus England und Deutschland (Governance in Initial Teacher Education: Perspectives on England and Germany). Wiesbaden: Springer VS.

Kuhlee, D. (2015). Studying and Learning in Initial Teacher Education in Germany in the Aftermath of the Bologna Reform Process: Ins ights into the Micro-Level Governance. In: Kuhlee, D., Buer, J. van \& Winch, C. (Hrsg.), Governance in der Lehrerausbildung: Analysen aus England und Deutschland (Governance in Initial Teacher Education: Perspectives on England and Germany) (pp. 169-189). Wiesbaden: Springer VS.

Kultusministerkonferenz (2001). Einigung der Kultusministerkonferenz mit den Lehrerverbänden über Konsequenzen aus PISA. Pressemitteilung der KMK vom 05.12.2001. Bonn: KMK. Abrufbar unter http://www.kmk.org/no_cache/presse-und-aktuelles/pm2001/einigung-mitlehrerverbaenden.html?sword_list[0]=konsequenzen\&sword_list[1]=pisa

Kultusministerkonferenz (2004). Standards für die Lehrerbildung: Bildungswissenschaften. Beschluss der KMK vom 16.12.2004. Bonn: KMK.

Kultusministerkonferenz (2007). Bildung für nachhaltige Entwicklung in der Schule. Empfehlung der Kultusministerkonferenz und der Deutschen UNESCO-Kommission vom 15.06.2007. Bonn: KMK.

Kultusministerkonferenz (2008). Ländergemeinsame inhaltliche Anforderungen für die Fachwissenschaften und Fachdidaktiken in der Lehrerbildung. Beschluss vom 16.10.2008 in der Fassung vom 08.12.2008. Bonn: KMK.

Kultusministerkonferenz (2009a). Empfehlung der Kultusministerkonferenz zur Stärkung der mathematischnaturwissenschaftlich-technischen Bildung. Beschluss der KMK vom 07.05.2009. Bonn: KMK. 
Kultusministerkonferenz (2009b). Konzeption der Kultusministerkonferenz zur Nutzung der Bildungsstandards für die Unterrichtsentwicklung. Beschluss der KMK vom 10.12.2009. Bonn: KMK.

Kultusministerkonferenz (2010). Ländergemeinsame inhaltliche Anforderungen für die Fachwissenschaften und Fachdidaktiken in der Lehrerbildung. Beschluss der KMK vom 16.10.2008 i.d.F. vom 16.09.2010. Bonn: KMK.

Kultusministerkonferenz (2011a). Lehrereinstellungsbedarf und Lehrereinstellungsangebot in der Bundesrepublik Deutschland. Modellrechnung 2010-2020. Juni 2011. Bonn \& Berlin: KMK

Kultusministerkonferenz (2011b). Handreichung für die Erarbeitung von Rahmenlehrplänen der Kultusministerkonferenz für den berufsbezogenen Unterricht in der Berufsschule und ihre Abstimmung mit Ausbildungsordnungen des Bundes für anerkannte Ausbildungsberufe. Sekretariat der Kultusministerkonferenz 23.09.2011. Berlin: KMK.

Kultusministerkonferenz (2013a). Gemeinsame Erklärung der Kultusministerkonferenz und der Organisation von Menschen mit Migrationshintergrund zur Bildungs- und Erziehungspartnerschaft von Schule und Eltern. Beschluss der KMK vom 10.10.2013. Bonn: KMK.

Kultusministerkonferenz (2013b). Regelungen und Verfahren zur Erhöhung der Mobilität und Qualität von Lehrkräften. Ländergemeinsame Umsetzungsrichtlinien für die Anpassung von Regelungen und Verfahren bei der Einstellung in Vorbereitungs- und Schuldienst sowie für die Anerkennung von Studien und Prüfungsleistungen in Studiengängen der Lehramtsausbildung. Beschluss der KMK vom 07.03.2013 i.d.F. vom 27.12.2013. Bonn: KMK.

Kultusministerkonferenz (2014). Sachstand in der Lehrerbildung (30.10.2014). Bonn: KMK.

Kultusministerkonferenz (2015). Lehrerbildung für eine Schule der Vielfalt. Gemeinsame Empfehlung von Hochschulrektorenkonferenz und Kultusministerkonferenz. Beschluss der KMK vom 12.03.2015 sowie Beschluss der HRK vom 18.03.2015. Bonn: KMK.

Kunter, M., Baumert, J., Blum, W., Klusmann, U., Krauss, S. \& Neubrand, M. (Hrsg.) (2011). Professionelle Kompetenz von Lehrkräften. Ergebnisse des Forschungsprogramms COACTIV. Münster: Waxmann.

Kurtz, T. (2009). Professionalität aus soziologischer Perspektive. In: Zlatkin-Troitschanskaia, O., Beck, K. u. a. (Hrsg.), Lehrprofessionalität. Bedingungen, Genese, Wirkungen und ihre Messung (pp. 45-54). Weinheim/Basel: Beltz.

Lauterbach, K. W. \& Schrappe, M. (2011). Gesundheitsökonomie, Qualitätsmanagement und Evidencebased Medicine. Stuttgart: Schattauer.

Lehmann, R. H., Peek, R., Gänsfuß, R., Lutkat, S., Mücke, S. \& Barth, I. (2000). QuaSUM Qualitätsuntersuchung an Schulen zum Unterricht in Mathematik. Ergebnisse einer repräsentativen Untersuchung im Land Brandenburg. Potsdam: Ministerium für Bildung, Jungend und Sport des Landes Brandenburg.

Lorenz, D. (1981). Wissenschaft zwischen Hochschulautonomie und Staatsintervention. Juristenzeitung, Nr. 4, 20. Februar 1981.

Maroy, C. \& Zanten, A., van (2009). Regulation and competition among schools in six European localities. Sociologie du travail, Heft 1, pp. 67-79.

Ministerium für Kultus, Jugend und Sport (2010). Handreichung zum Schulpraxissemester. Baden-Württemberg. Abrufbar unter www.lehrer.uni-karsruhe.de/za242/PS/ PSHandreichung.pdf.

Monitor Lehrerbildung (2015). Aufgaben der Zentren für Lehrerbildung. Gütersloh: Bertelsmann et al. Abrufbar unter http://www.monitor-lehrerbildung.de/web/diagramm/hsfrage 59 
Die ,neue' Lehrerbildung in Deutschland. Vom Praxisschock zur aufgeklärten Aneignung professionellen Handelns?...

Müller, K. (2010). Das Praxisjahr in der Lehrerbildung. Empirische Befunde zur Wirksamkeit studienintegrierter Langzeitpraktika. Bad Heilbrunn: Klinkhardt.

Müller, S., Pietsch, M. \& Bos, W. (Hrsg.) (2011). Schulinspektion in Deutschland. Eine Zwischenbilanz aus empirischer Sicht. Münster: Waxmann.

Nölle, K. (2002). Problem der Form und des Erwerbs unterrichtsrelevanten Wissens. Zeitschrift für Pädagogik, Heft 1, pp. 48-67.

Oelkers, J. (2007). Kompetenz und Professionalität: Neue Wege in der Lehrerbildung. Vortrag im Staatlichen Seminar für Didaktik und Lehrerbildung (GHS) Lörrach am 6. März 2007.

Offenberg, E. \& Walke, J. (2013). Die Reform der Praxisphasen in der Ersten Phase der Lehrerbildung. Eine qualitative Dokumentenanalyse. Essen: Stifterverband für die Deutsche Wissenschaft.

Popham, W. J. (2001). Teaching to the test. Educational Leadership, Vol. 58, No. 6, pp. 16-20.

Radtke, F.-O. (2004). Der Eigensinn pädagogischer Professionalität jenseits von Innovationshoffnungen und Effizienzerwartungen. Übergangene Einsichten aus der Wissensverwendungsforschung für die Organisation der universitären Lehrerbildung. In: Koch-Priewe, B., Kolbe, F. U. \& Wildt, J. (Hrsg.), Grundlagenforschung und mikrodidaktische Reformansätze zur Lehrerbildung (pp. 99-149). Bad Heilbrunn: Klinkhardt.

Reinhoffer, B. \& Dörr, G. (2008): Zur Wirksamkeit Schulpraktischer Studien. In: Rotermund, M., Dörr, G., Bodensohn, R. (Hrsg.), Bologna verändert die Lehrerbildung. Auswirkungen der Hochschulreform (pp. 10-31). Leipzig: Leipziger Univ.-Verlag.

Reinisch, V. (2009). „Lehrprofessionalität" als theoretischer Term. Eine begriffssystematische Analyse. In: Zlatkin-Troitschanskaia, O., Beck, K. u. a. (Hrsg.), Lehrprofessionalität. Bedingungen, Genese, Wirkungen und ihre Messung (pp. 33-43). Weinheim/Basel: Beltz.

Riese, J. (2009). Professionelles Wissen und professionelle Handlungskompetenz von (angehenden) Physiklehrkräften. Berlin: Logos.

Rischke, M., Bönsch, C. \& Müller, U. (2013). Praxisbezug in der Lehrerbildung - je mehr, desto besser? Sonderpublikation aus dem Projekt Monitor Lehrerbildung. Gütersloh: Zentrum für Hochschulentwicklung. Abrufbar unter http://2015.monitorlehrerbildung.de/export/sites/default/.content/Downloads/Monitor_Lehrerbildung_Praxisbezug_10_ 2013.pdf .

Rogal, U. (2008). Hochschulautonomie und Zielvereinbarungen. Neue Instrumente der Hochschulsteuerung und ihre Vereinbarkeit mit der akademischen Selbstverwaltung. Hamburg: Kovac.

Rolff, H.-G. (2011). Das System des UQM im Überblick. In: Rolff, H.-G. (Hrsg.), Qualität mit System. Eine Praxisanleitung zum Unterrichtsbezogenen Qualitätsmanagement (UQM). Neuwied: Carl Link.

Rolff, H.-G. (2014). Zur Rolle von Peer Reviews in der Schulentwicklung und im Qualitätsmanagement. In: Pfeiffer, M. (Hrsg.), Schulqualität und Schulentwicklung. Theorien, Analysen und Potenziale (pp. 208227). Münster: Waxmann.

Roters, B., Schneider, R., Koch-Priewe, B., Thiele, J. \& Wildt, J. (Hrsg.) (2009). Forschendes Lernen im Lehramtsstudium. Hochschuldidaktik, Professionalisierung, Kompetenzentwicklung. Bad Heilbrunn: Klinkhardt.

Rürup, M. (2005). Der Föderalismus als institutionelle Rahmenbedingung im deutschen Bildungswesen Perspektiven der Bildungspolitikforschung. Deutsches Institut für Internationale Pädagogische Forschung (DIPF). peDOCS (Open Access Erziehungswissenschaften). 
Rürup, M. (2007). Innovationswege im deutschen Bildungssystem. Die Verbreitung der Idee „Schulautonomie im Ländervergleich“. Wiesbaden: VS Verlag für Sozialwissenschaften.

Schmitt, C. (2009): Kompetenzentwicklung der Jenaer Lehramtsstudierenden im betreuten Blockpraktikum. Diplomarbeit. Jena: Friedrich Schiller-Universität Jena.

Schmitt, C. \& Gröschner, A. (2010). Belastungsempfinden und Kompetenzentwicklung von Lehramtsstudierenden im modularisierten Studium - Empirische Studien am Beispiel des Jenaer Modells. Power Point Präsentation. Bielefeld (09.02.2010).

Schneider, R. \& Wildt, J. (2009). Forschendes Lernen in Praxisstudien - Wechsel eines Leitmotivs. In: Roters, B., Schneider, R. et al. (Hrsg.), Forschendes Lernen im Lehramtsstudium. Hochschuldidaktik, Professionalisierung, Kompetenzentwicklung (pp. 8-36). Bad Heilbrunn: Klinkhardt.

Schratz, M. \& Steiner-Löfler, U. (1999). Die Lernende Schule. Arbeitsbuch pädagogische Schulentwicklung. Weinheim/Basel: Beltz.

Schumacher, K. \& Lind, G. (2000). Praxisbezug im Lehramtsstudium - Bericht einer Befragung von Konstanzer LehrerInnen und Lehramtsstudierenden. Forschungsbericht. Konstanz: Universität Konstanz.

Senatsverwaltung für Bildung, Jugend und Wissenschaft (2012). Ausbildung von Lehrkräften in Berlin. Empfehlungen der Expertenkommission Lehrerbildung. Berlin.

Senatsverwaltung für Bildung, Jugend und Wissenschaft (2014). Handbuch Vorbereitungsdienst. Materialien für den reformierten Berliner Vorbereitungsdienst. Berlin.

Shulman, L. S. (1986). Those Who Understand: Knowledge Growth in Teaching. Educational Researcher, Heft 2, pp. 4-14.

Shulman, L. S. (1987). Knowledge and teaching: Foundations of the New Reform. Harvard Educational Review, Heft 6, pp. 1-22.

Sprigade, A. (2015). The Labour Market for Teachers in England: Teachers and Recruitment Difficulties in Schools. In: Kuhlee, D., Buer, J. van \& Winch, C. (Hrsg.), Governance in der Lehrerausbildung: Analysen aus England und Deutschland (Governance in Initial Teacher Education: Perspectives on England and Germany) (pp. 99-110). Wiesbaden: Springer VS.

Stender, J. (2011). Berufsbildung in der Bundesrepublik Deutschland 1: Strukturprobleme und Ordnungsprinzipien des dualen Systems. Stuttgart: Hirzel.

Terhart, E. (1990). Pädagogisches Wissen in subjektiven Theorien: das Beispiel Lehrer. In: Drerup, H. \& Terhart, E. (Hrsg.), Erkenntnis und Gestaltung (pp. 117-143). Weinheim: Deutscher Studien Verlag.

Terhart, E. (2006). Standards und Kompetenzen in der Lehrerausbildung. In: Hilligius, A. H. \& Rinkens, H.D. (Hrsg.), Standards und Kompetenz - neue Qualität in der Lehrerausbildung? Neue Ansätze und Erfahrungen in nationaler und internationaler Perspektive. Paderborner Lehrerausbildungszentrum der Universität Paderborn, Band 11 (pp. 29-42). Berlin: LIT-Verlag.

Timmermann, D. \& Windschild, T. (1996). Implementierung von Qualitätsgrundsätzen und -maßstäben in der betrieblichen Bildung. In: Timmermann, D. (Hrsg.), Qualitätsmanagement in der betrieblichen Bildung (pp. 79-90). Bielefeld: Bertelsmann.

Tittle, C. K. (2006). Assessment of teacher learning and development. In: Alexander, P. A. \& Winne, P. H. (Ed.), Handbook of Educational Psychology, $2^{\text {nd }}$ ed., Vol. 41 (pp. 953-980). Mahwah, NJ: Lawrence Erlbaum Associates. 
Die ,neue' Lehrerbildung in Deutschland. Vom Praxisschock zur aufgeklärten Aneignung professionellen Handelns?...

Treiber, B. \& Weinert, F. E. (Hrsg.) (1982). Lehr-Lern-Forschung. Ein Überblick in Einzeldarstellungen. München et al.: Urban \& Schwarzenberg.

Ulich, K. (1996). Beruf Lehrer/in. Arbeitsbelastungen - Beziehungskonflikte - Zufriedenheit. Weinheim: Beltz.

Vogel, T. (2011). Zum Theorie-Praxis-Verhältnis in der Lehrerbildung als Übergangsproblem. In: Diehl, T., Krüger, J. \& Vogel, T. (Hrsg.), bwp@ Spezial 5 - Hochschultage Berufliche Bildung 2011, Workshop 14, pp. 1-14. (http://www.bwpat.de/ht2011/ws14/vogel_ws14-ht2011.pdf, abgerufen am 11.07.2015).

Voss, T., Kunter, M., Seiz, J., Hoehne, V. \& Baumert, J. (2014). Die Bedeutung des pädagogischpsychologischen Wissens von angehenden Lehrkräften für die Unterrichtsqualität. Zeitschrift für Pädagogik, 60. Jahrgang, Heft 2, pp. 184-201.

Wagner, C. (2011). Führung und Qualitätsmanagement in beruflichen Schulen - Triangulative Fallstudien zum Führungsverständnis und Führungshandeln einzelschulischer Führungskräfte. Frankfurt/M.: Lang.

Ward, B. E. (1970). A survey of microteaching in NCATE-accredited secondary education programs (R\&D Memo No. 70). Standford, California: Standford Center for Research and Development in Teaching.

Wenglein, S., Bauer, J., Heininger, S. \& Prenzel, M. (2015). Kompetenz angehender Lehrkräfte zum Argumentieren mit Evidenz: Erhöht ein Training von Heuristiken die Argumentationsqualität? Unterrichtswissenschaft, 43. Jahrgang, Heft 3, 209-224.

Werth, W. (1985). Die Vermittlung von Theorie und Praxis an den Preussischen Pädagogischen Akademien 1926 -1933 dargestellt am Beispiel der Pädagogischen Akademie Halle/Saale (1930-1933). Frankfurt a. M.: dipa.

Weyland, U. \& Wittmann, E. (2011). Expertise. Praxissemester im Rahmen der Lehrerbildung. 1. Phase an hessischen Hochschulen. Berlin: Dipf.

Wideen, M., Mayer-Smith, J. \& Moon, B. (1998). A critical analysis of the research in learning to teach: Making the case for an ecological perspective on inquiry. Review of Educational Research, 68, pp. 130-178.

Wisemann, D., Knight, S. \& Cooner, D. (2004). Becoming a Teacher in a Field-Based Setting: An Introduction to Education and Classrooms. 3rd Edition. Boston: Wadsworth Publishing Co Inc.

Wittmann, E. (2007). Explizite und implizite Veränderung von Verwaltungskonzepten in der "neuen" Schule - Analysen zum Berliner Schulgesetz. In: Buer, J. van \& Wagner, C. (Hrsg.), Qualität von Schule. Ein kritisches Handbuch (pp. 201-216). Frankfurt a. M.: Lang.

Zeichner, K. M. (2010). Rethinking the Connections between Campus Courses and Field Experiences in College- and University-Based Teacher Education. Journal of Teacher Education, Vol. 61, No. 1-2, pp. 89-99.

Zeichner, K. M. \& Tabachnick, B. R. (1981). Are the effects of university teacher education "washed out" by school experiences? Journal of Teacher Education, Vol. 32, No. 2, 7-11.

Zeuch, N. \& Souvignier, E. (2015). Zentrale Facetten wissenschaftlichen Denkens bei Lehramtsstudierenden - Entwicklung eines neuen Instruments und Identifikation von latenten Profilen. Unterrichtswissenschaft, 43. Jahrgang, Heft 3, 245-262.

Zinn, B. (2013). Überzeugungen zu Wissen und Wissenserwerb von Auszubildenden. Münster: Waxmann. 
Zlatkin-Troitschanskaia, O. (2007). Steuerungsfähigkeit des öffentlichen Schulwesens versus Steuerbarkeit der Schule - Paradigmenwechsel? In: Buer, J. van \& Wagner, C. (Hrsg.), Qualität von Schule. Ein kritisches Handbuch (pp. 67-81). Frankfurt a. M.: Lang.

Fecha de recepción / Received: 08/06/2015

Fecha de aceptación / Accepted: 30/06/2015 